\title{
ships4sst
}

\section{Sentinel-3 SLSTR SST Validation using a Fiducial Reference Measurements (FRM) Service}

W. Wimmer, T. Nightingale, J. Høyer, R. Wilson, H. Kelliher, C. Donlon 


\section{Outline}

- Introduction

- Data sources

- Validation methods

- Validation Results

- Reprocessed data 2016 -2018 - global

- QI results - reprocessed data 2016-2018 - global

- D3, D2, N3, N2 results for the reprocessed data 2016-2018 - global

- Conclusion

- Appendix

- NRT validation results, 2018 


\section{Data}

- SLSTR

- Reprocessed data: 2016-2018 v006 r1i1

- NRT data 2018 onwards

- FRM

- Radiometer data from ships4sst

- The MDB files are produced by Felyx

- SLSTR L1b and L2 data within 400x400 pixels of matchup

- L2R data within 6hrs of matchup

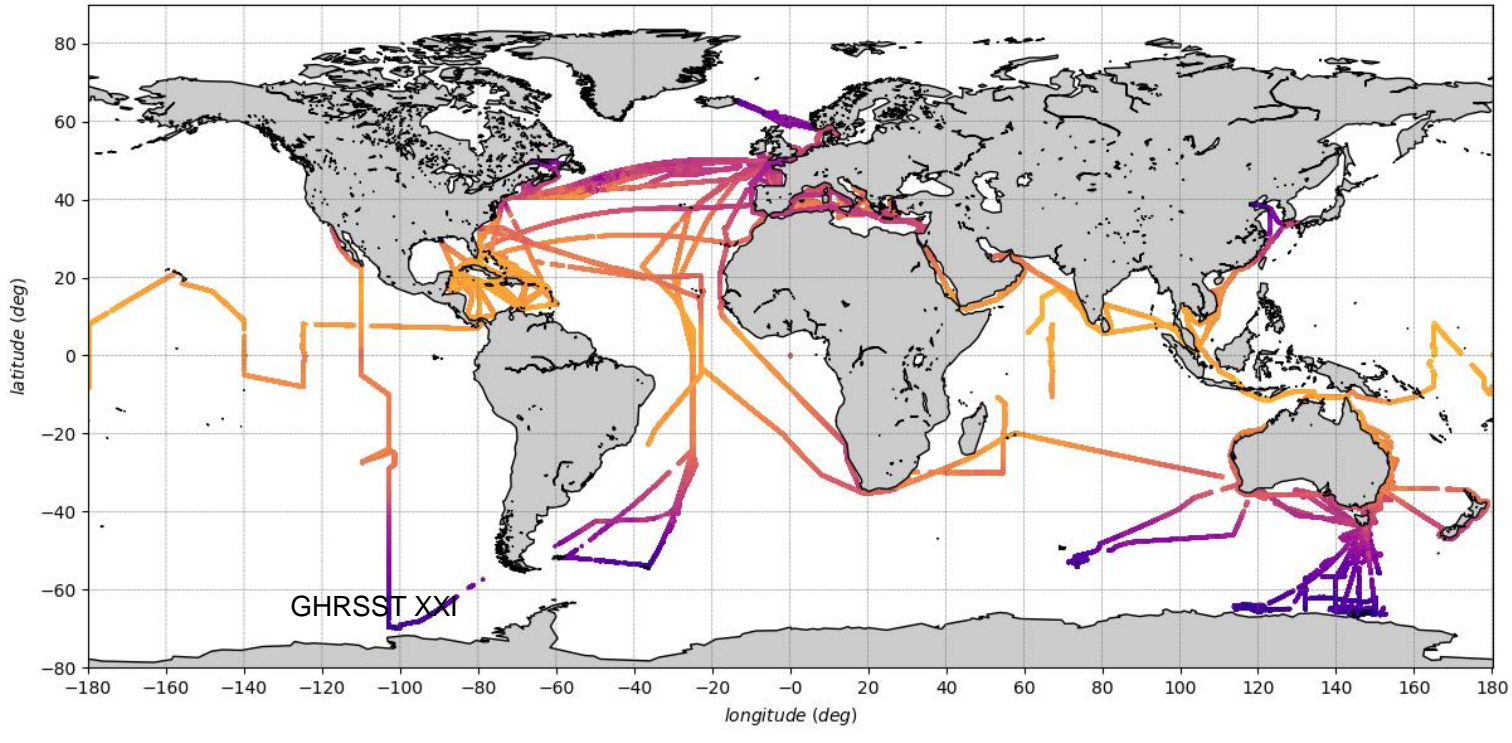




\section{Validation methods}

- MDB files (as produced by felyx) are reprocessed

- allow multiple matches per match-up location (overpass)

- Validate WST, D3, D2, N3, N2 and all GHRSST CV levels $(0,3,4,5)$

- follow the Wimmer et.al. 2012 approach.

- Quality indicator method developed for AATSR used on SLSTR

- Wimmer et. al. 2012:

- Matchup grades

\begin{tabular}{|r|r|r|}
\hline grade & temporal (h) & spatial (km) \\
\hline 1 & 0.5 & 1 \\
\hline $2 \mathrm{a}$ & 0.5 & 20 \\
\hline $2 \mathrm{~b}$ & 2.0 & 1 \\
\hline 3 & 2.0 & 20 \\
\hline 4 & 6.0 & 25 \\
\hline
\end{tabular}




\section{Validation results - global}

- WST - August 2016 - April 2018, grade 2b

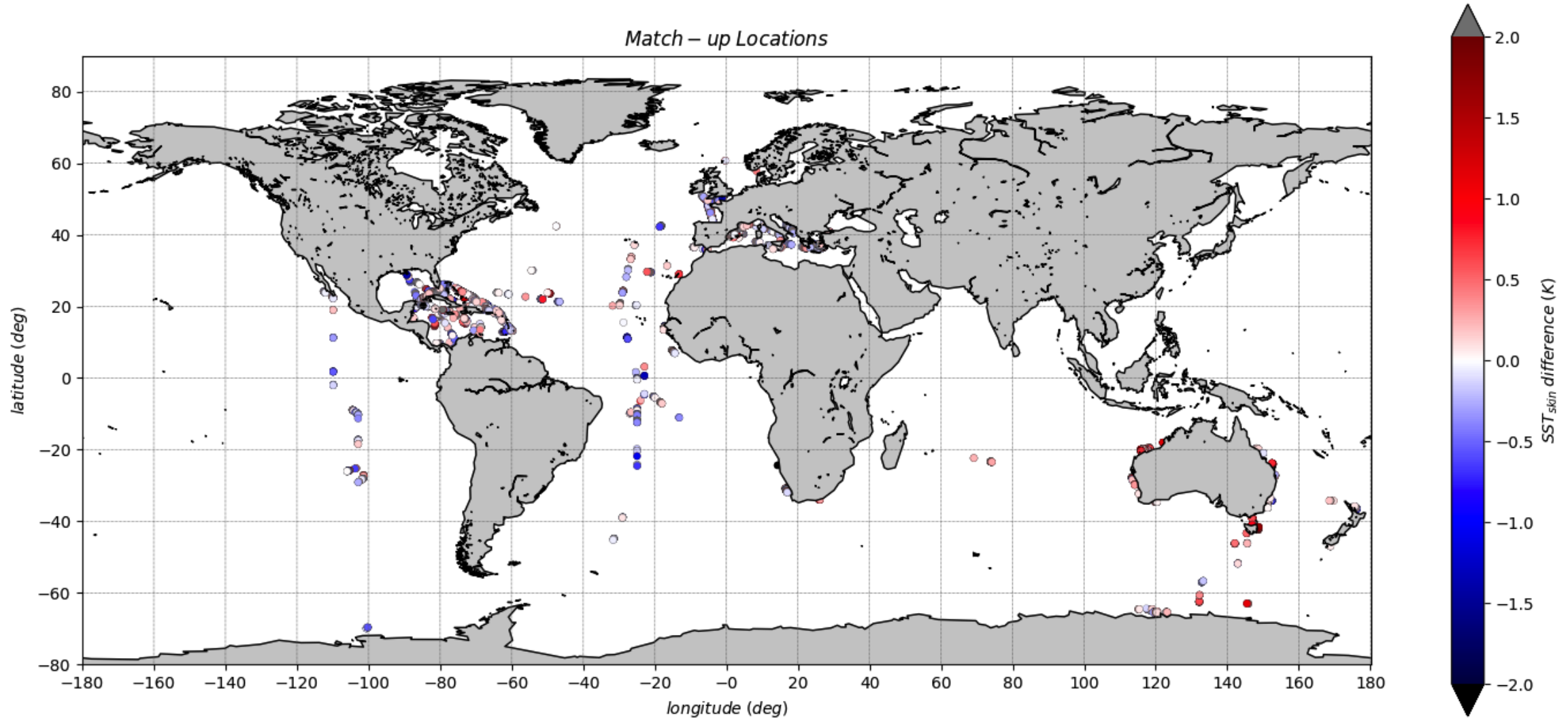

\section{- esa}




\section{Validation results - histograms - all}

\section{$2016-2018$}

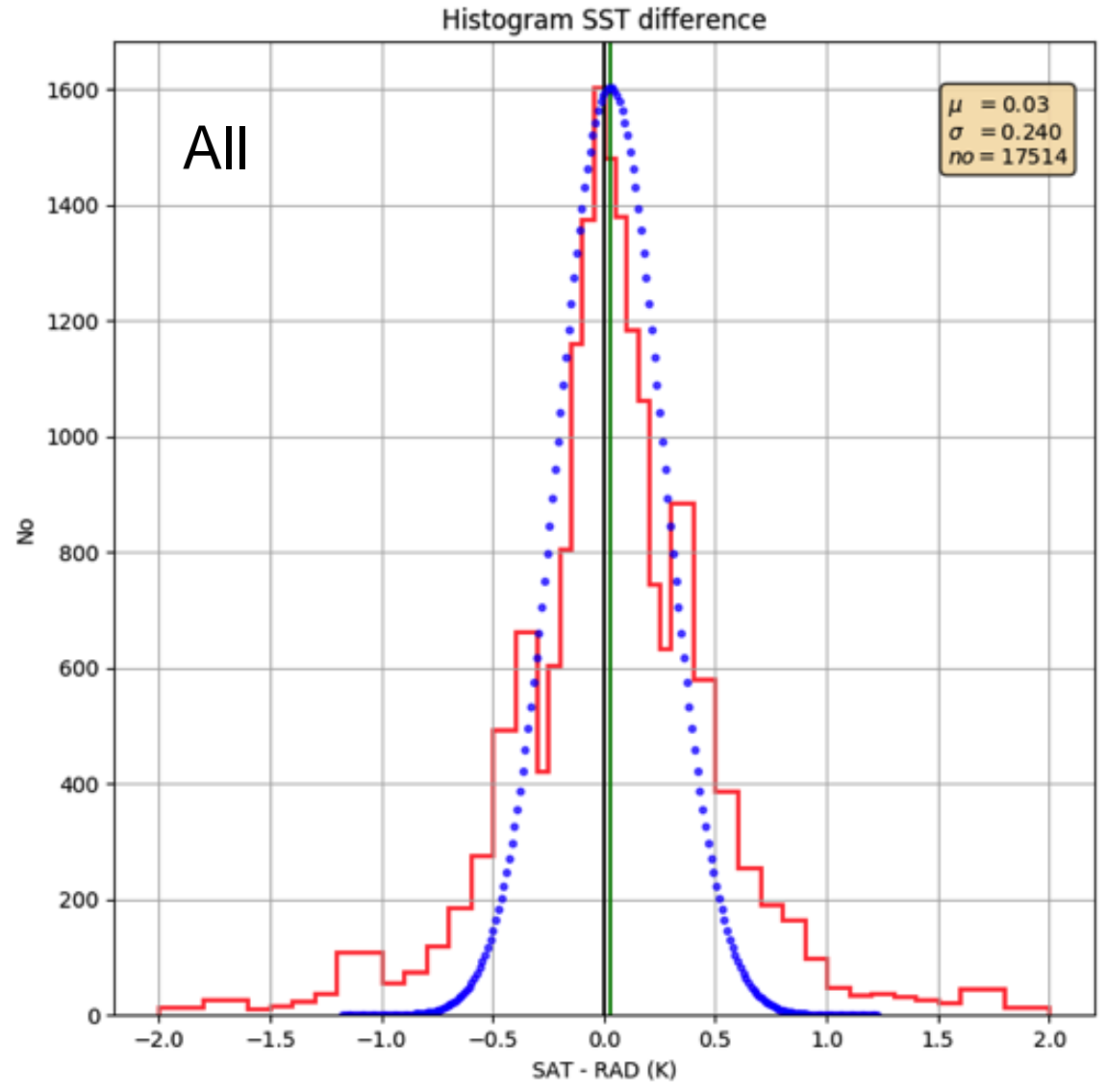

s45str111, all, sstdiff_sst_wst, grade 2b, all, ghrsst-all, qi-all - HuberT processed 20200507 (c) 2020 ISAR team - v5.2
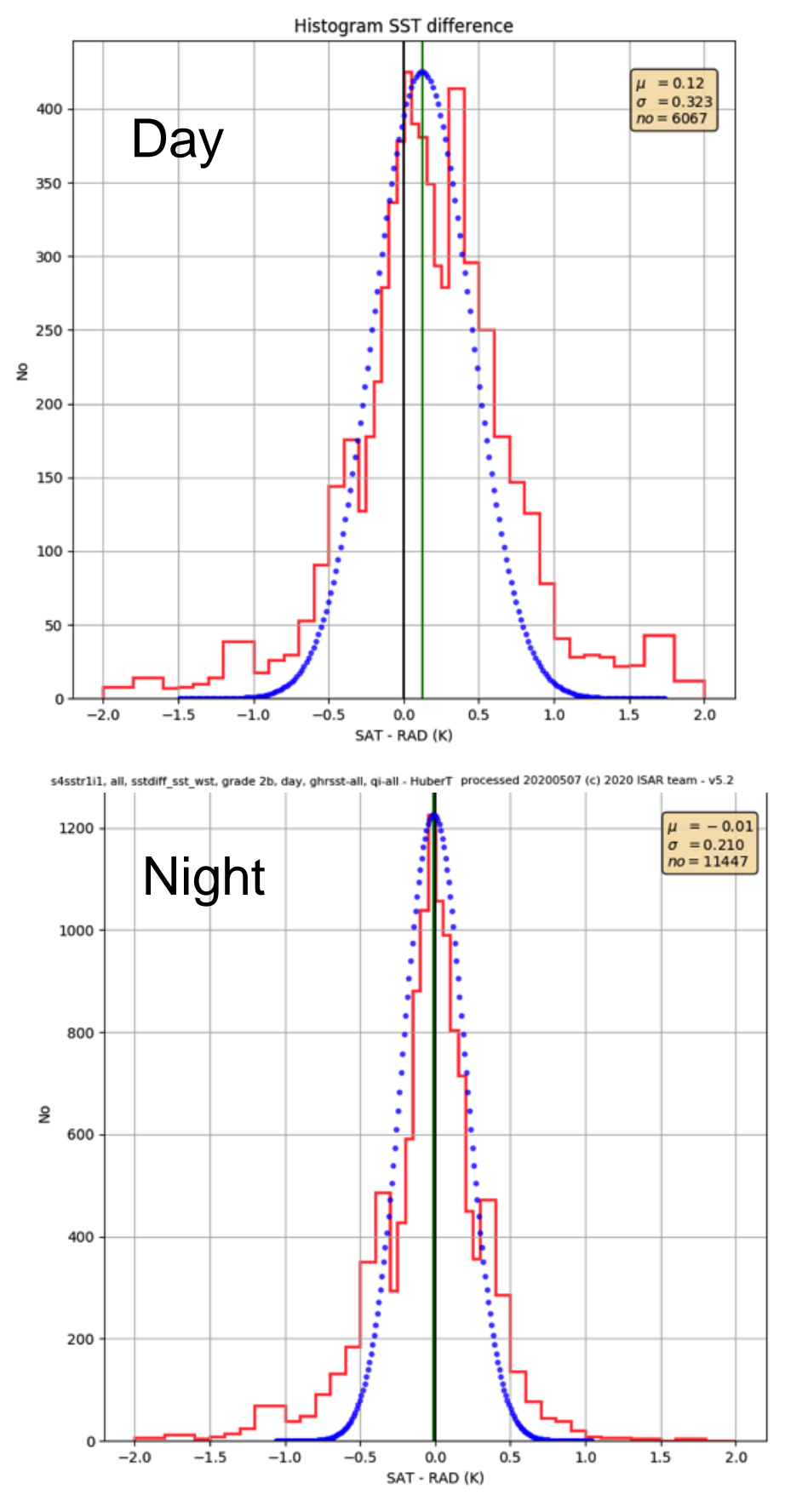


\section{Histograms - CV5}

\section{$2016-2018$}

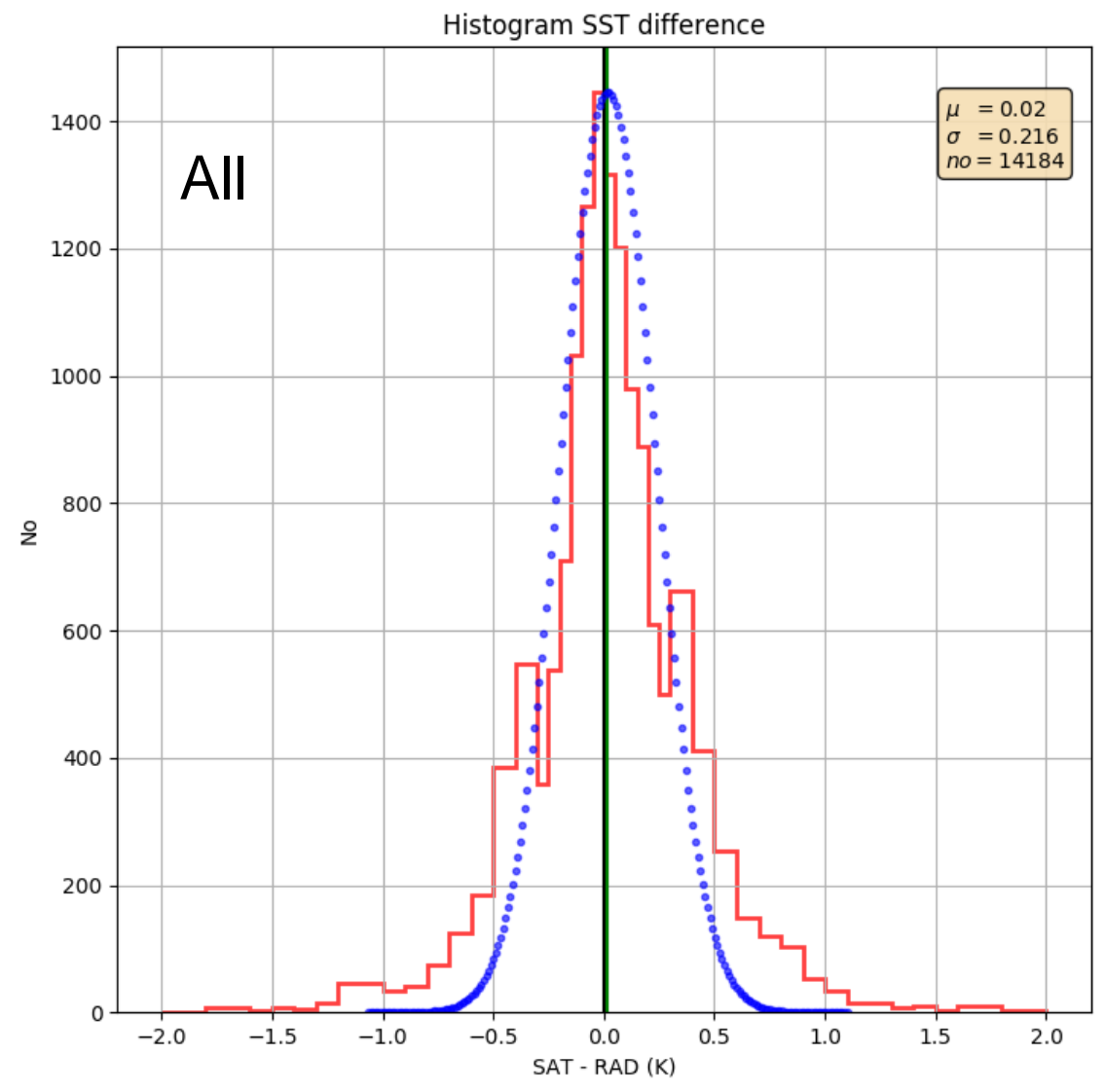

54sstr1i1, all, sstdiff_sst_wst, grade 2b, all, ghrsst-5, qi-all - HuberT processed 20200507 (c) 2020 ISAR team - v5.2

\section{- cesa}
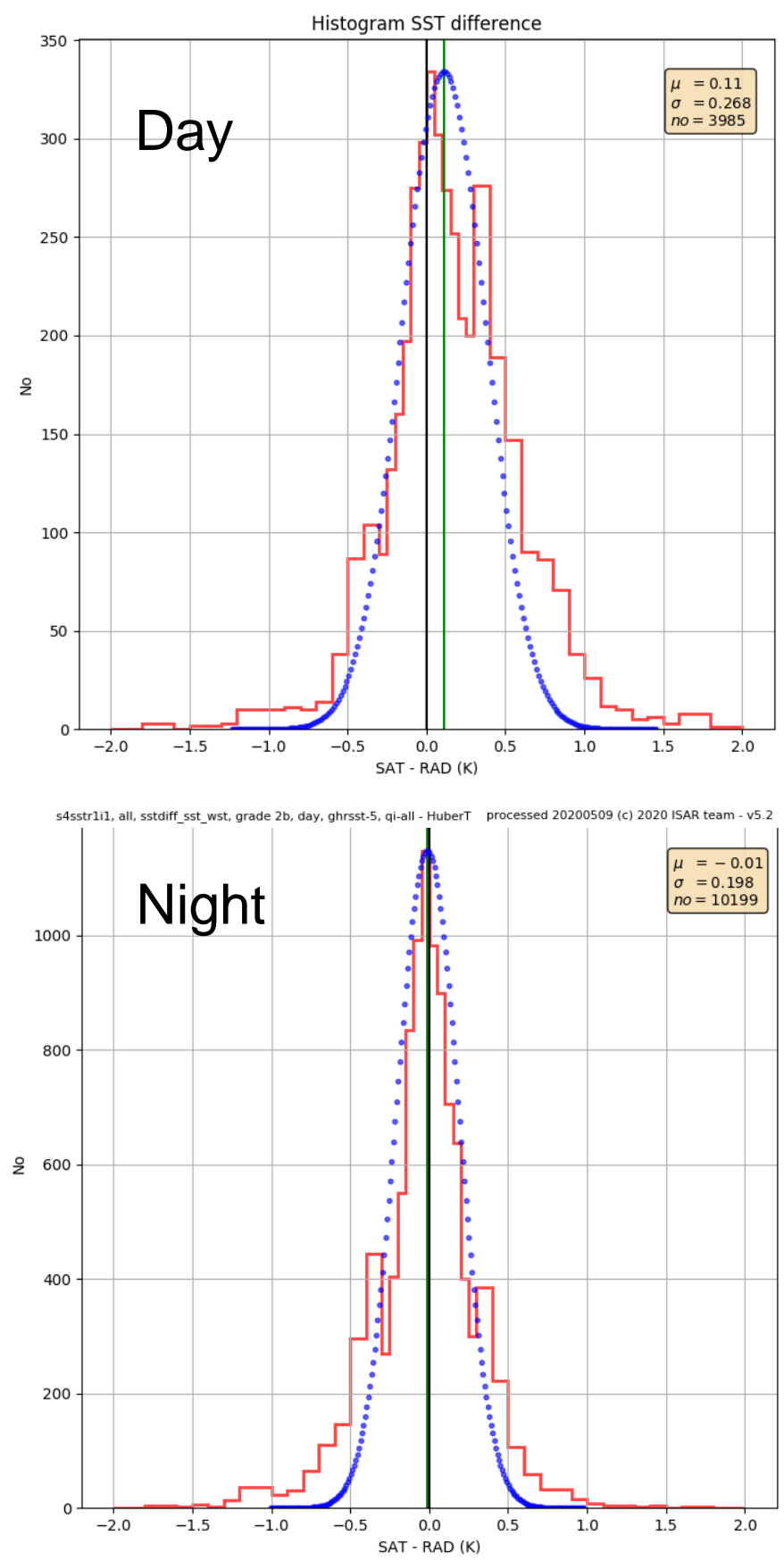


\section{Validation scatter - all}

\section{$2016-2018$}

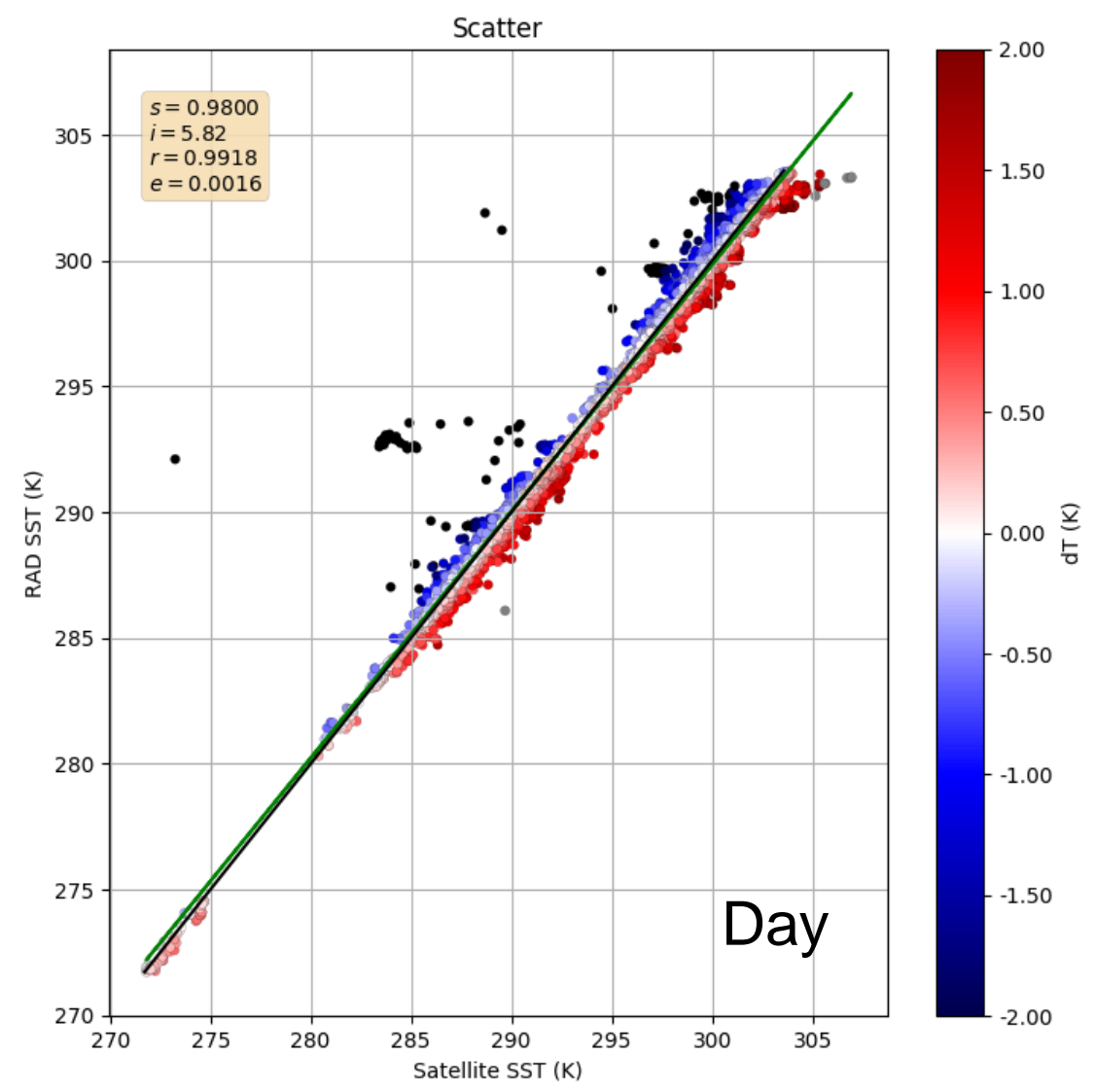

54sstr1i1, all, sstdiff _sst wst, grade 2b, day, ghrsst-all, qi-all - HuberT processed 20200507 (c) 2020 ISAR team - v5.2

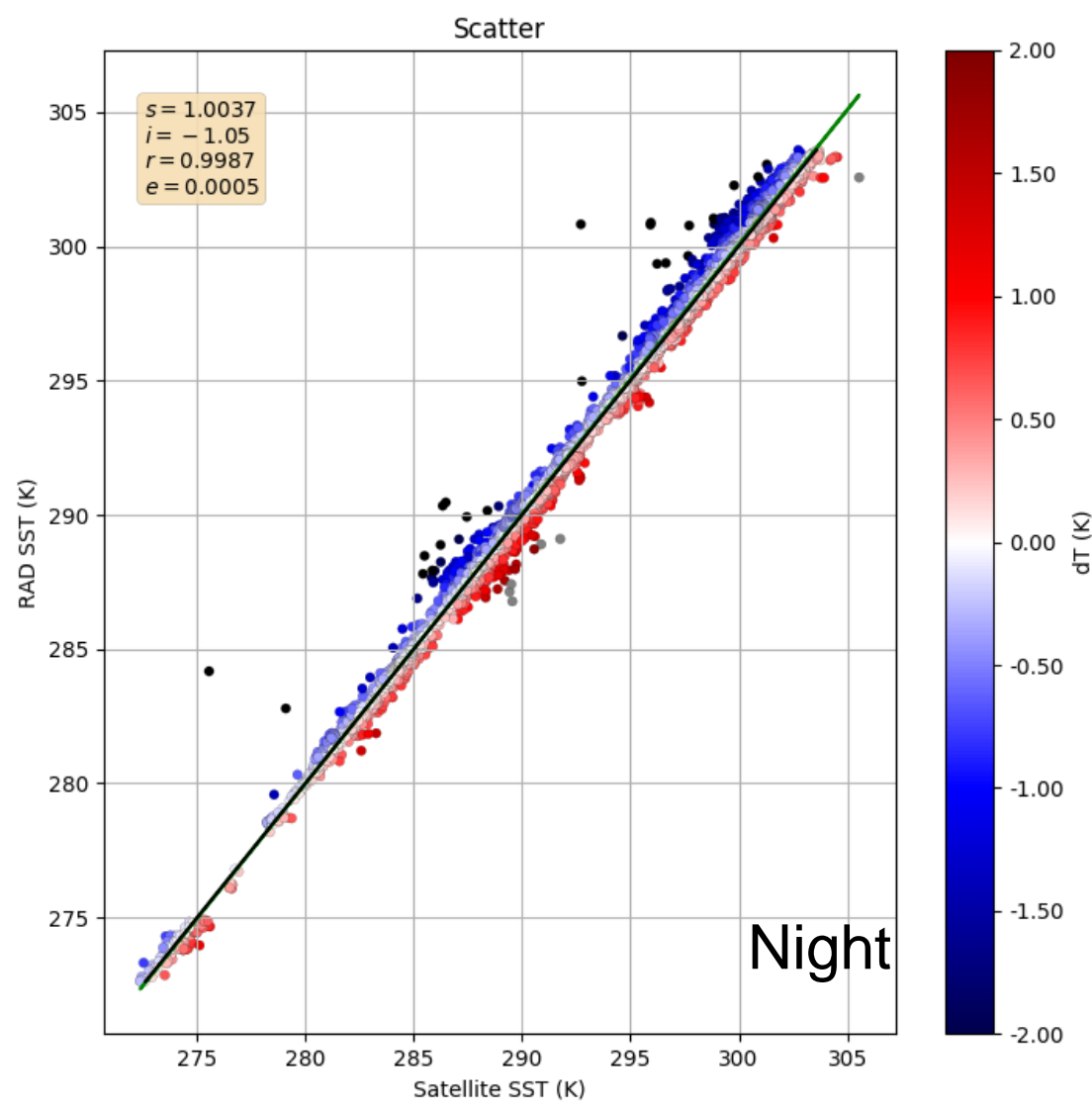

54sstr1i1, all, sstdiff_sst_wst, grade 2b, night, ghrsst-all, qi-all - HuberTprocessed 20200507 (c) 2020 ISAR team - v5.2

\section{Cesa}

\section{RAL Space Southampton}

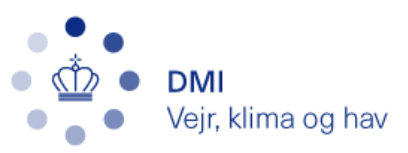




\section{Validation - scatter, CV 5}

\section{$2016-2018$}

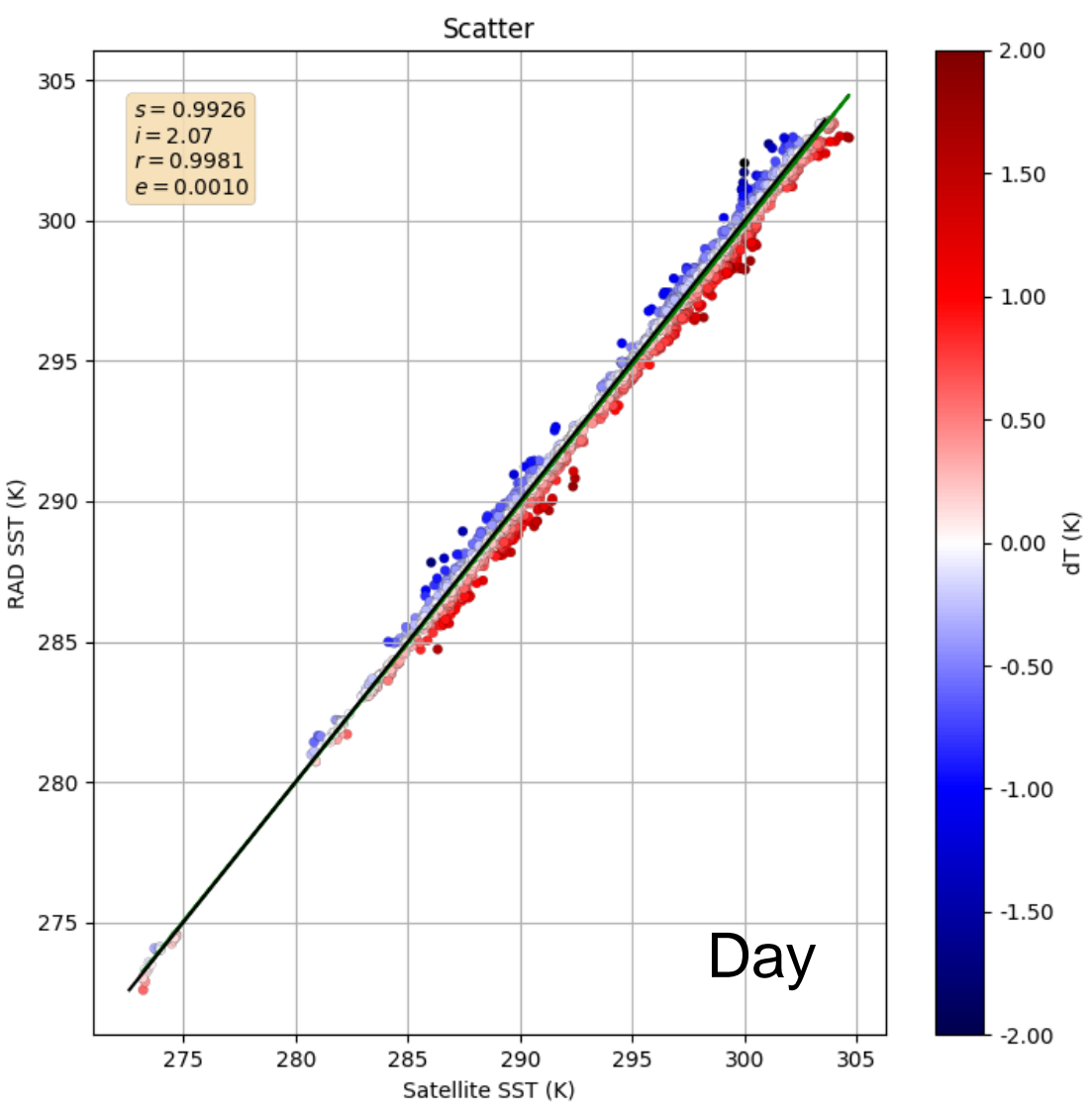

54sstr1i1, all, sstdiff_sst_wst, grade 2b, day, ghrsst-5, gi-all - HuberT processed 20200507 (c) 2020 ISAR team - v5.2

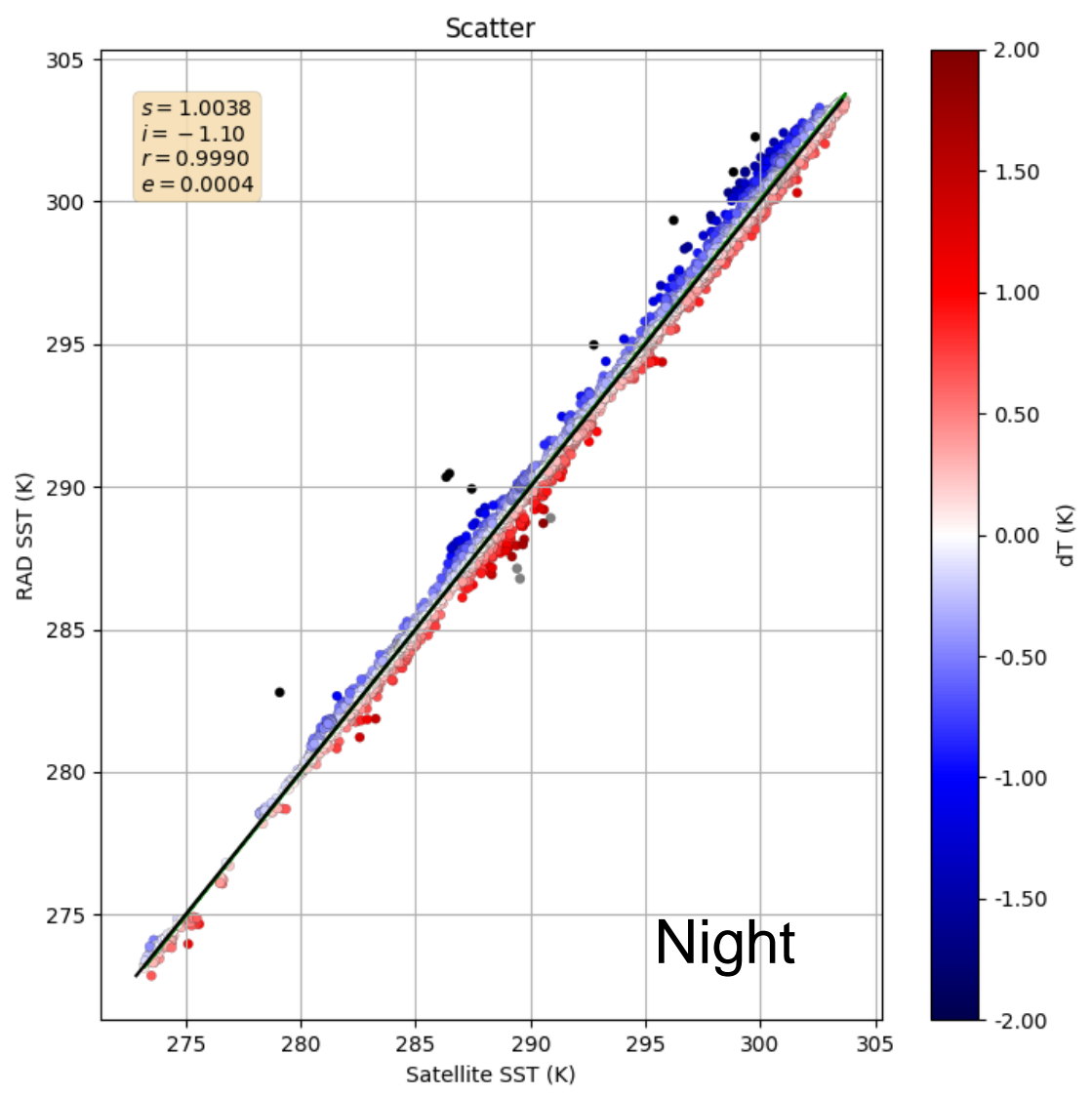

54sstr1i1, all, sstdiff sst wst, grade 2b, night, ghrsst-5, qi-all - HuberT processed 20200507 (c) 2020 ISAR team - v5 2

\section{Cesa}

\section{RAL Space Southampton}

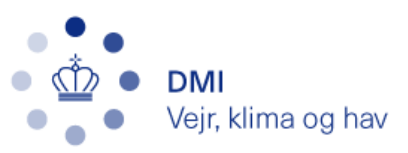


Validation results - statistics

- 2016 -2018: All data

\begin{tabular}{|r|r|r|r|r|r|r|}
\hline \multicolumn{1}{|l|}{ lay } \\
\hline \multicolumn{1}{|l|}{ Mrade } & \multicolumn{1}{|l|}{ MDiff } & RSD & No & Overpass & Min Temp & Max Temp \\
\hline 1 & 0.12 & 0.28 & 1521 & 193 & 271.95 & 304.30 \\
\hline $2 \mathrm{a}$ & 0.11 & 0.40 & 4888 & 367 & 270.48 & 307.29 \\
\hline $\mathbf{2 b}$ & $\mathbf{0 . 1 2}$ & $\mathbf{0 . 3 2}$ & $\mathbf{6 0 6 7}$ & $\mathbf{3 1 8}$ & $\mathbf{2 7 1 . 8 0}$ & $\mathbf{3 0 6 . 9 5}$ \\
\hline 3 & 0.12 & 0.43 & 18663 & 458 & 270.20 & 307.34 \\
\hline 4 & 0.09 & 0.50 & 54823 & 643 & 270.20 & 307.48 \\
\hline
\end{tabular}

\begin{tabular}{|r|r|r|r|r|r|r|}
\hline \multicolumn{70}{|l|}{ WST } \\
\hline \multicolumn{10}{|l|}{ ALL } \\
\hline Grade & \multicolumn{1}{|l|}{ MDiff } & \multicolumn{1}{l|}{ RSD } & \multicolumn{1}{l|}{ No } & Overpass & Min Temp & Max Temp \\
\hline 1 & 0.03 & 0.22 & 4518 & 542 & 271.95 & 304.30 \\
\hline $2 \mathrm{a}$ & 0.04 & 0.32 & 12548 & 967 & 270.48 & 307.29 \\
\hline $\mathbf{2 b}$ & $\mathbf{0 . 0 3}$ & $\mathbf{0 . 2 4}$ & $\mathbf{1 7 5 1 4}$ & $\mathbf{8 1 9}$ & $\mathbf{2 7 1 . 8 0}$ & $\mathbf{3 0 6 . 9 5}$ \\
\hline 3 & 0.04 & 0.33 & 48138 & 1161 & 270.20 & 307.34 \\
\hline 4 & 0.03 & 0.38 & 132206 & 1475 & 270.20 & 307.48 \\
\hline
\end{tabular}

\begin{tabular}{|c|c|c|c|c|c|c|}
\hline \multicolumn{7}{|l|}{$\begin{array}{l}\text { Night } \\
\text { Nigh }\end{array}$} \\
\hline Grade & MDiff & RSD & No & Overpass & Min Temp & Max Temp \\
\hline 1 & -0.01 & 0.20 & 2997 & 349 & 272.38 & 304.28 \\
\hline $2 a$ & 0.00 & 0.27 & 7660 & 600 & 271.80 & 304.68 \\
\hline $2 b$ & -0.01 & 0.21 & 11447 & 501 & 272.38 & 305.55 \\
\hline 3 & 0.00 & 0.28 & 29475 & 703 & 271.30 & 305.55 \\
\hline 4 & 0.00 & 0.31 & 77383 & 832 & 271.30 & 305.55 \\
\hline
\end{tabular}




\section{Validation results - statistics}

- 2016 -2018: CV5

\begin{tabular}{|r|r|r|r|r|r|r|}
\hline \multicolumn{1}{|l|}{ Day } \\
\hline \multicolumn{1}{|l|}{ Grade } & \multicolumn{1}{l|}{ MDiff } & \multicolumn{1}{l|}{ RSD } & No & Overpass & Min Temp & Max Temp \\
\hline 1 & 0.10 & 0.24 & 1046 & 139 & 273.25 & 303.90 \\
\hline $2 \mathrm{a}$ & 0.15 & 0.32 & 2821 & 248 & 273.16 & 303.94 \\
\hline $\mathbf{2 b}$ & $\mathbf{0 . 1 1}$ & $\mathbf{0 . 2 7}$ & $\mathbf{3 9 8 5}$ & $\mathbf{2 3 3}$ & $\mathbf{2 7 3 . 1 9}$ & $\mathbf{3 0 4 . 6 6}$ \\
\hline 3 & 0.16 & 0.35 & 10565 & 332 & 273.16 & 304.66 \\
\hline 4 & 0.13 & 0.42 & 31538 & 505 & 273.15 & 304.66 \\
\hline
\end{tabular}

\begin{tabular}{|r|r|r|r|r|r|r|}
\hline \multicolumn{70}{|l|}{ WST } \\
\hline \multicolumn{10}{|l|}{ ALL } \\
\hline Grade & \multicolumn{1}{|l|}{ MDiff } & \multicolumn{1}{l|}{ RSD } & No & Overpass & Min Temp & Max Temp \\
\hline 1 & 0.02 & 0.20 & 3696 & 456 & 273.20 & 303.90 \\
\hline $2 \mathrm{a}$ & 0.05 & 0.27 & 9375 & 783 & 273.16 & 303.94 \\
\hline $\mathbf{2 b}$ & $\mathbf{0 . 0 2}$ & $\mathbf{0 . 2 2}$ & $\mathbf{1 4 1 8 4}$ & $\mathbf{6 9 0}$ & $\mathbf{2 7 3 . 1 9}$ & $\mathbf{3 0 4 . 6 6}$ \\
\hline 3 & 0.04 & 0.28 & 35744 & 971 & 273.15 & 304.78 \\
\hline 4 & 0.04 & 0.33 & 97827 & 1289 & 273.15 & 305.05 \\
\hline
\end{tabular}

\begin{tabular}{|r|r|r|r|r|r|r|}
\hline \multicolumn{1}{|l|}{ Night } \\
\hline \multicolumn{1}{|l|}{ Grade } & \multicolumn{1}{|l|}{ MDiff } & \multicolumn{1}{l|}{ RSD } & No & Overpass & Min Temp & Max Temp \\
\hline 1 & -0.01 & 0.19 & 2650 & 317 & 273.20 & 303.71 \\
\hline $2 \mathrm{a}$ & 0.01 & 0.25 & 6554 & 535 & 273.16 & 303.71 \\
\hline $\mathbf{2 b}$ & $-\mathbf{0 . 0 1}$ & $\mathbf{0 . 2 0}$ & $\mathbf{1 0 1 9 9}$ & $\mathbf{4 5 7}$ & $\mathbf{2 7 3 . 2 0}$ & $\mathbf{3 0 3 . 7 1}$ \\
\hline 3 & 0.00 & 0.26 & 25179 & 639 & 273.15 & 304.78 \\
\hline 4 & 0.01 & 0.29 & 66289 & 784 & 273.15 & 305.05 \\
\hline
\end{tabular}




\section{Validation results - QI}

- Estimateing match-up uncertainty :

- $\sigma=\sigma_{\mathrm{S}}+\sigma_{\mathrm{T}}+\sigma_{\mathrm{M}}+\sigma_{\mathrm{P}}+\sigma_{\mathrm{Z}}$

- $\sigma=\sigma_{\mathrm{S}}+\sigma_{\mathrm{T}}+\left(\sigma_{\mathrm{PS}}+\sigma_{\mathrm{PR}}\right)$

- Reprocessed data - August 2016 - April 2018

- Five indicators:

- Satellite variability - de-trended 9x9 field around match, weighted with chi2 to allow for cloudy pixels

- Satellite trend - 2d trend

- Radiometer variability de-trended track data, $2 \mathrm{~h}$ either side of match, weighted with chi2 to allow for missing data.

- Radiometer Trend

- Radiometer Sky BT

- Not everybody provides this data

- Only run on grade 4 (QI 0 is grade 4)

- Uses 4 levels $(0,3,4,5)$ 


\section{Validation results - QI histograms}

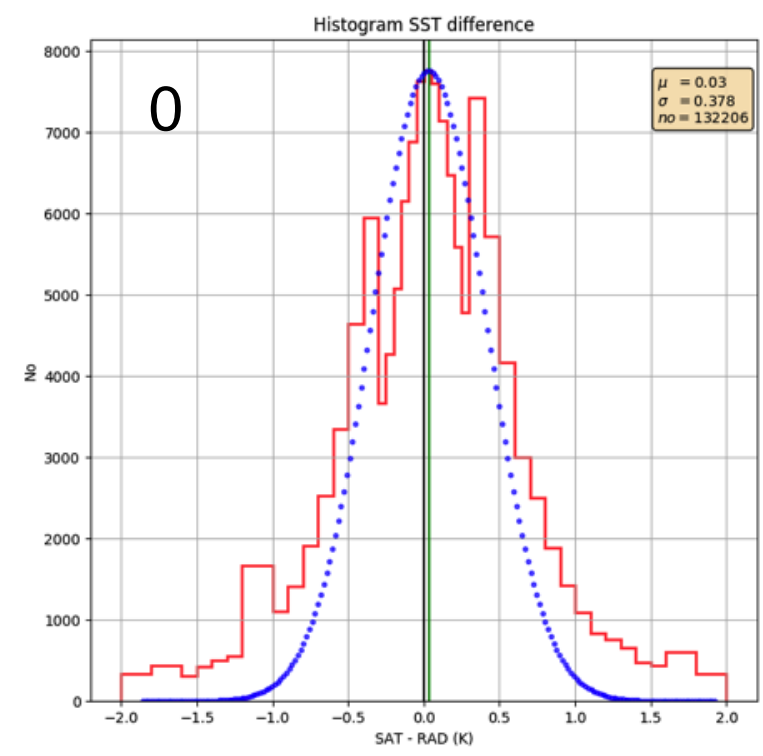

ships4sst: FRM4SST

Cesa
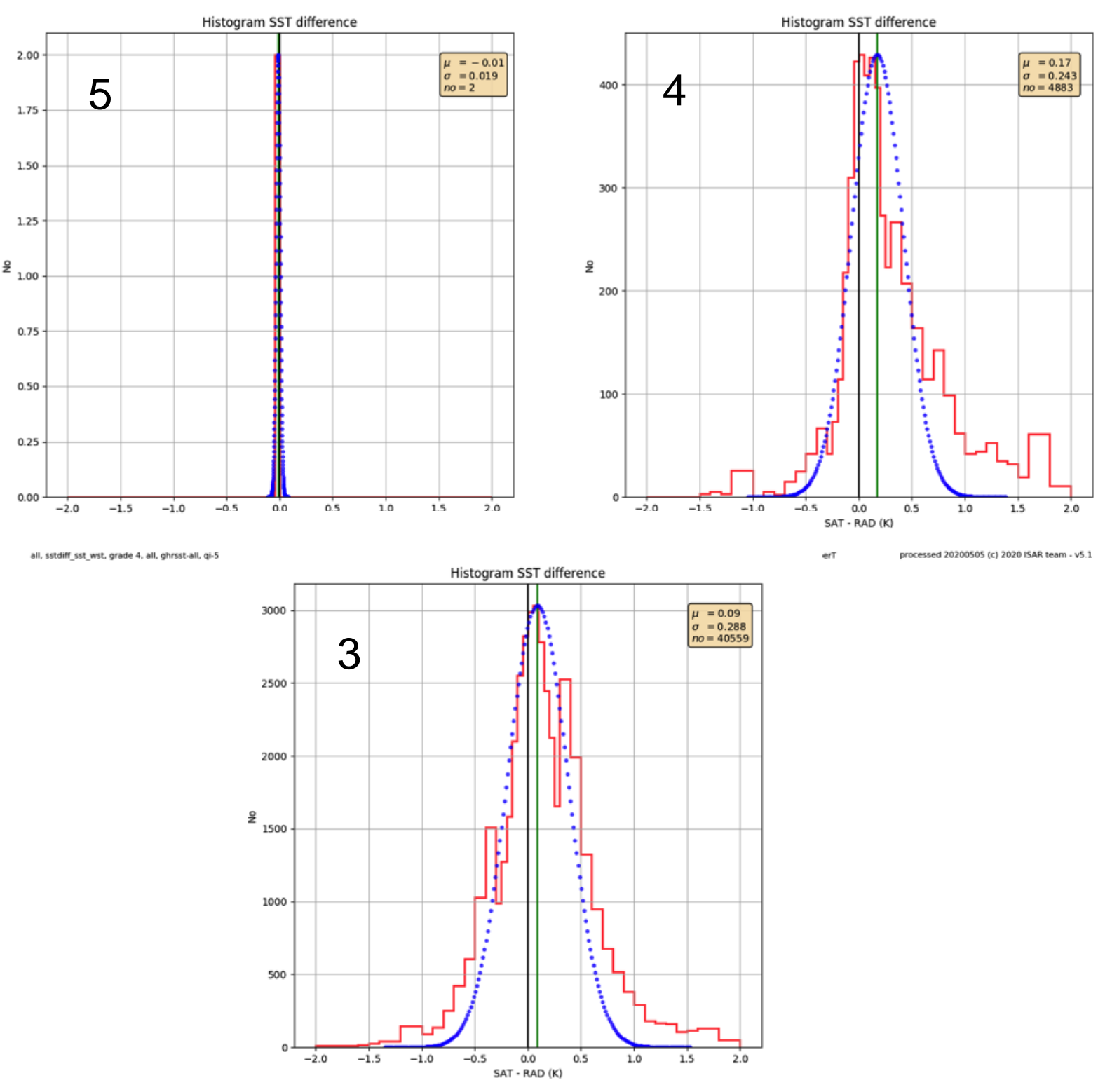

GHRSST XXI

02 June 2020

Page 14

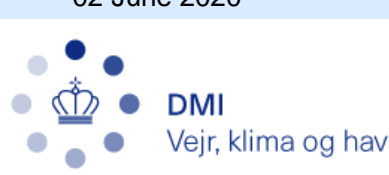


Histograms QI

\section{- esa}
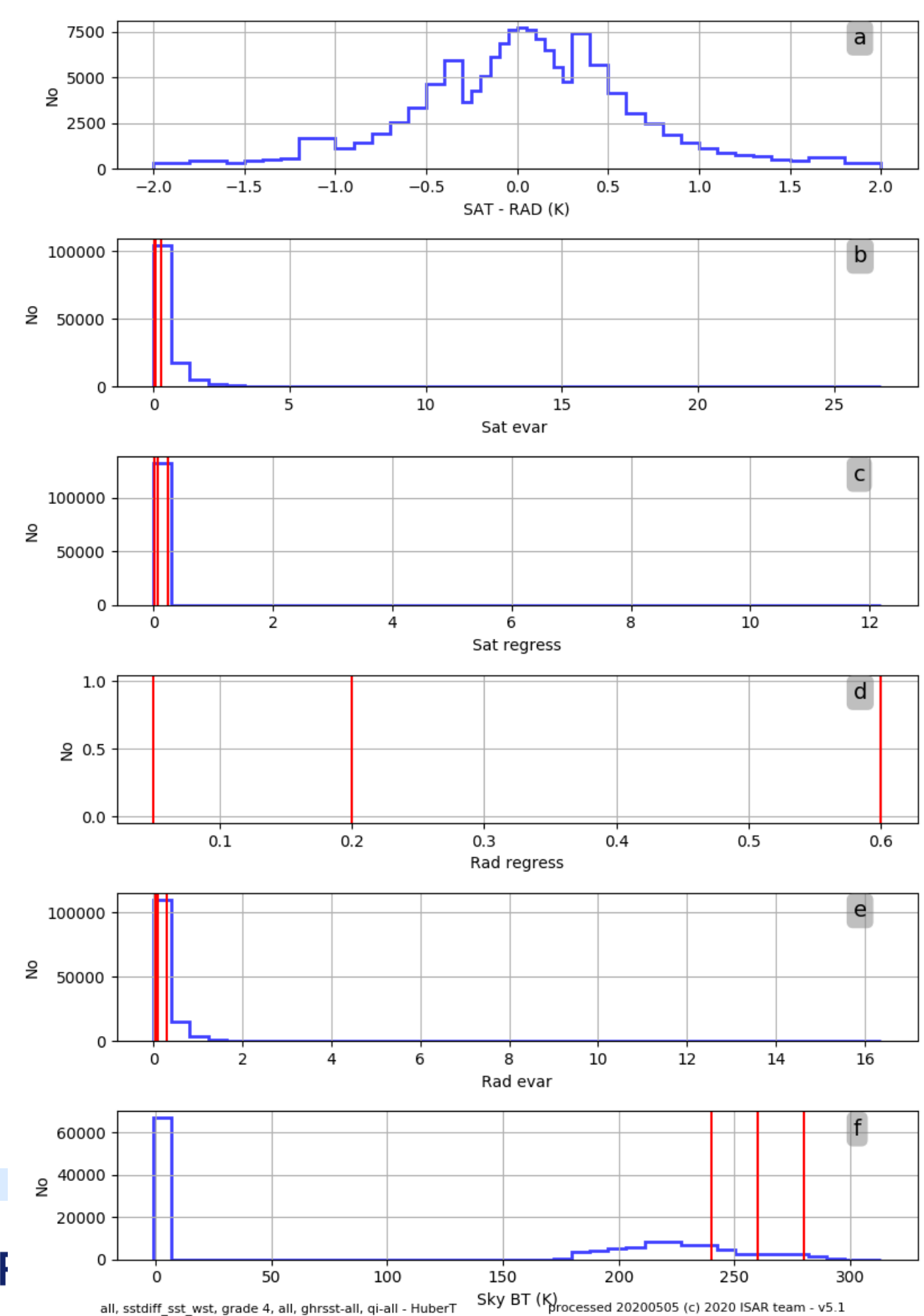

Page 15 Space Connexions 
Sat Evar

\section{Validation scatter - QI}
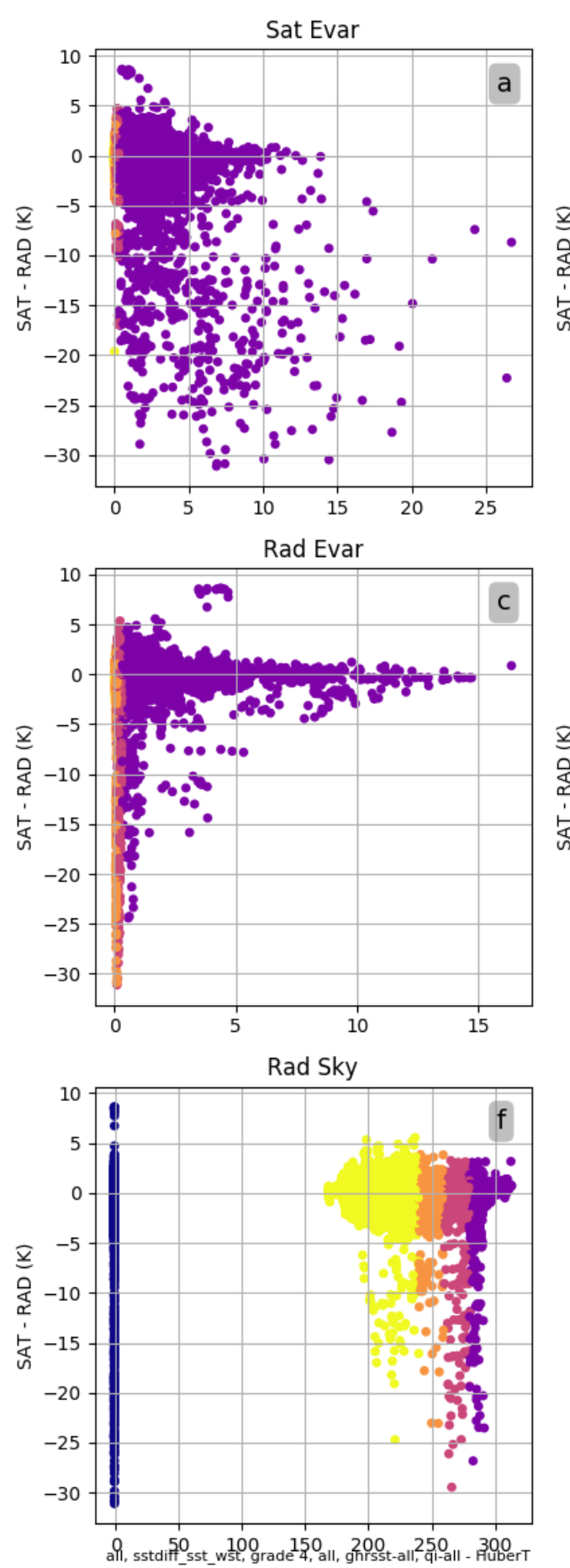

ships4sst: FRM4SST

\section{- Cesa}

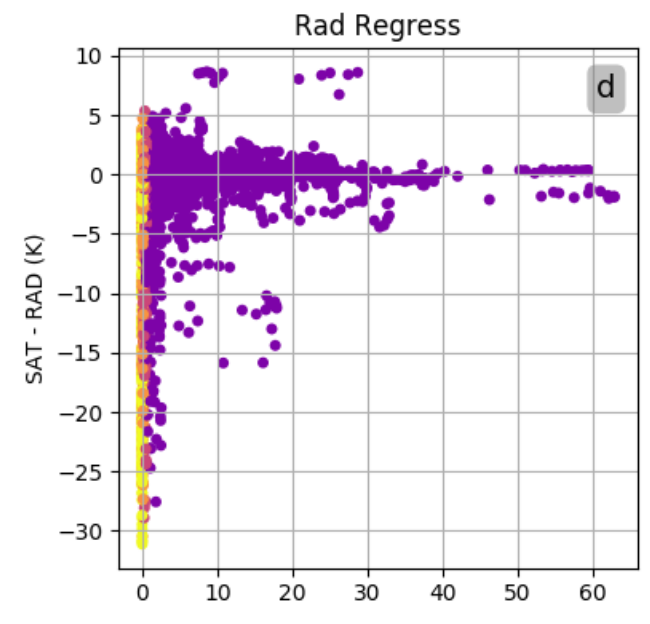

Sat Regress
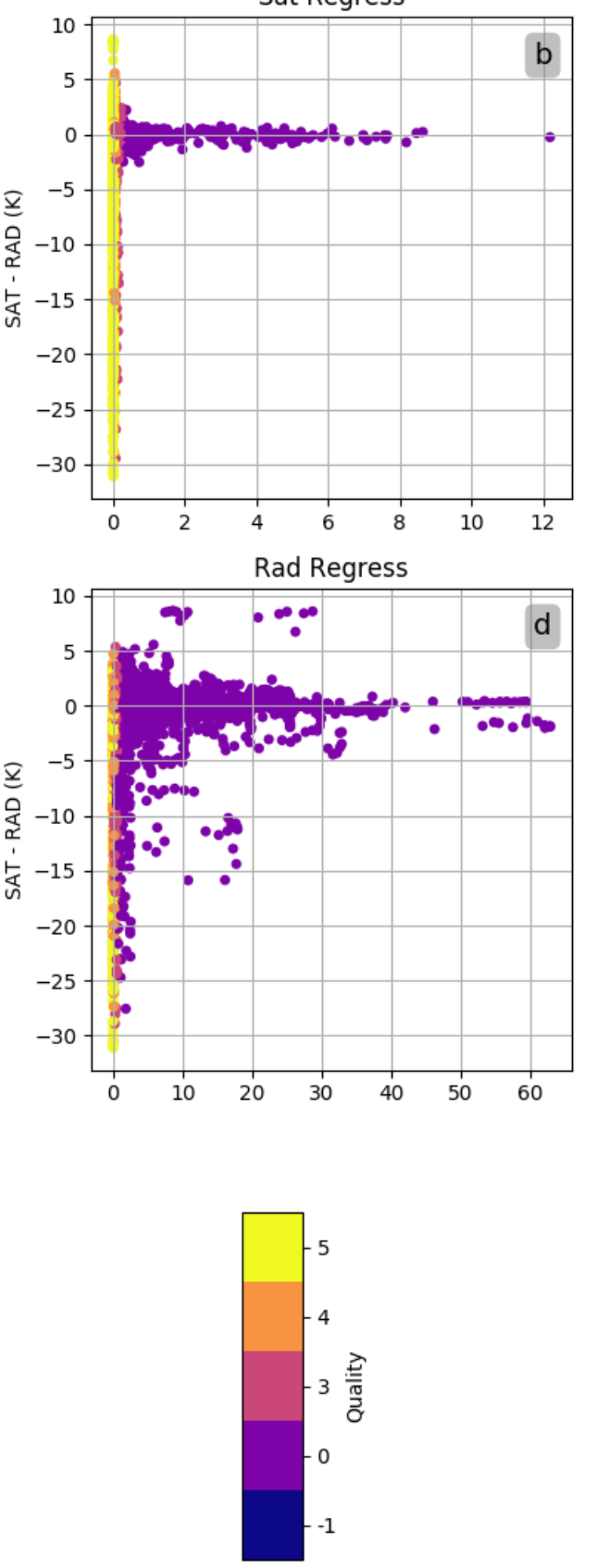

Page 16 


\section{Validation - Q}

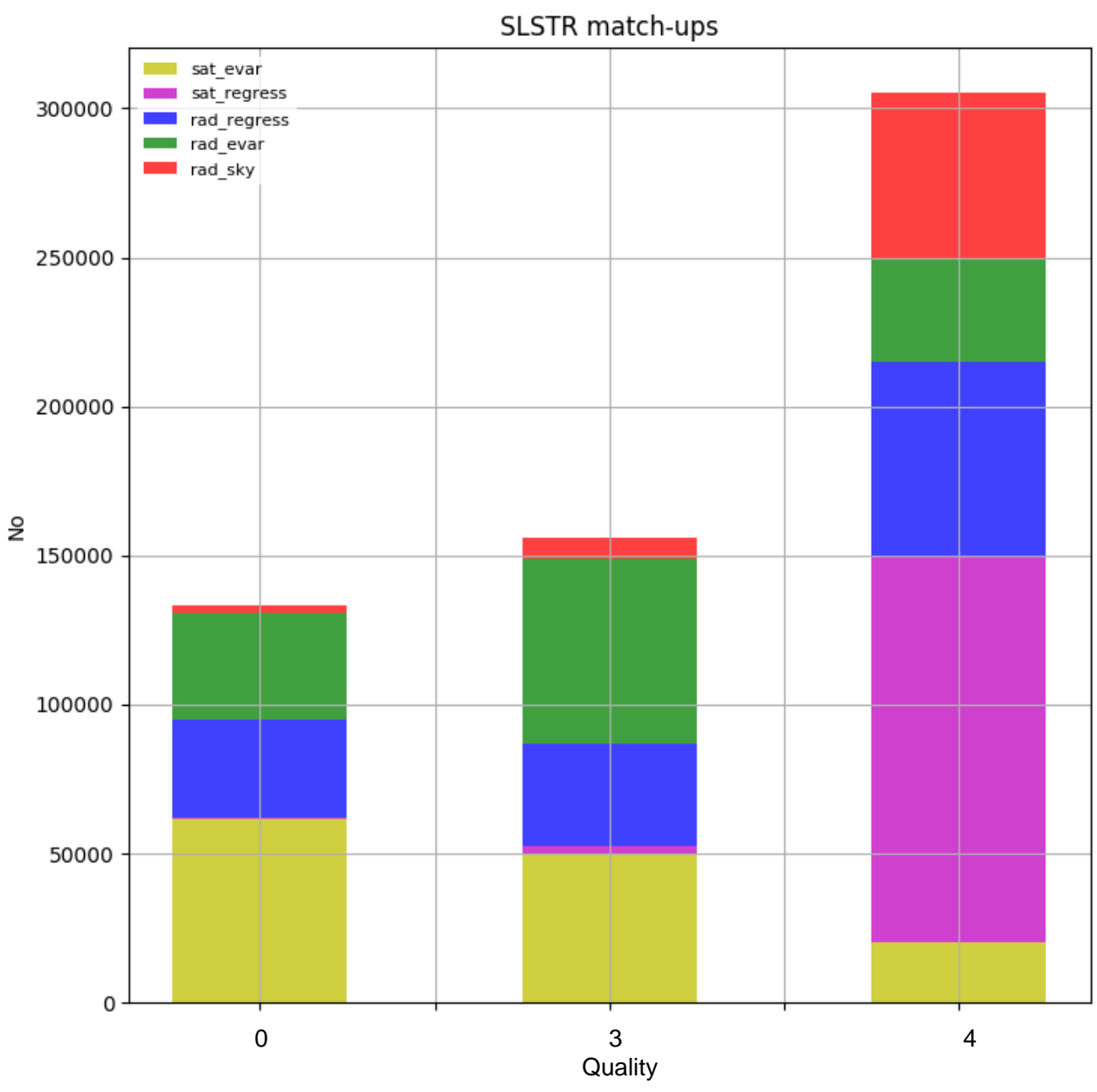

all, sstdiff_sst wst, grade 4, all, ghrsst-all, qi-all - HuberT

processed 20200505 (c) 2020 ISAR team - v5.1 


\section{Validation results - statistics}

- 2016- 2018 - QI

\begin{tabular}{|r|r|r|r|r|r|r|}
\hline Day & \multicolumn{9}{|l|}{} \\
\hline QI & \multicolumn{1}{|l|}{ MDiff } & \multicolumn{1}{l|}{ SSD } & No & Overpass & Min Temp & Max Temp \\
\hline 0 & 0.09 & 0.50 & 54823 & 643 & 270.20 & 307.48 \\
\hline 3 & 0.17 & 0.40 & 17244 & 401 & 271.78 & 304.24 \\
\hline 4 & 0.37 & 0.49 & 2056 & 117 & 273.65 & 304.07 \\
\hline 5 & \multicolumn{7}{|l}{} \\
\hline
\end{tabular}

\begin{tabular}{|c|c|c|c|c|c|c|}
\hline \multicolumn{7}{|l|}{ WST } \\
\hline \multicolumn{7}{|l|}{ All } \\
\hline QI & MDiff & RSD & No & Overpass & Min Temp & Max Temp \\
\hline 0 & 0.03 & 0.38 & 132206 & 1475 & 270.20 & 307.48 \\
\hline 3 & 0.09 & 0.29 & 40559 & 994 & 271.65 & 304.54 \\
\hline 4 & 0.17 & 0.24 & 4883 & 278 & 273.65 & 304.07 \\
\hline 5 & -0.01 & 0.02 & 2 & 1 & 296.79 & 296.79 \\
\hline
\end{tabular}

\begin{tabular}{|c|c|c|c|c|c|c|}
\hline \multicolumn{7}{|l|}{ Night } \\
\hline QI & MDiff & RSD & No & Overpass & Min Temp & Max Temp \\
\hline 0 & 0.00 & 0.31 & 77383 & 832 & 271.30 & 305.55 \\
\hline 3 & 0.04 & 0.23 & 23315 & 593 & 271.65 & 304.54 \\
\hline 4 & 0.09 & 0.17 & 2827 & 161 & 274.12 & 303.50 \\
\hline 5 & -0.01 & 0.02 & 2 & 1 & 296.79 & 296.79 \\
\hline
\end{tabular}




\section{Validation results - D3,D2,N3,N2 - all}

\section{- 2016 - 2018, all, CV5, D3, N3 excluded in day time}

D3

\begin{tabular}{|c|c|c|c|c|c|c|c|c|c|c|c|c|}
\hline \multicolumn{6}{|l|}{ Night } & \multicolumn{7}{|l|}{ N3 } \\
\hline Grade & MDiff & RSD & No & Overpass & Min Temp & Night & & & & & & \\
\hline 1 & -0.01 & 0.18 & 1339 & 317 & 273.20 & Grade & MDiff & RSD & No & Overpass & Min Temp & Max Temp \\
\hline $2 \mathrm{a}$ & 0.02 & 0.24 & 3051 & 535 & 272.97 & 1 & 0.02 & 0.17 & 1772 & 317 & 273.36 & 303.75 \\
\hline $2 \mathrm{~b}$ & -0.01 & 0.19 & 4775 & 457 & 273.20 & $2 a$ & 0.00 & 0.24 & 4535 & 535 & 273.00 & 303.75 \\
\hline 3 & 0.02 & 0.27 & 11846 & 639 & 272.97 & $2 \mathrm{~b}$ & 0.01 & 0.18 & 6739 & 457 & 273.36 & 303.75 \\
\hline 4 & 0.02 & 0.31 & 31362 & 784 & 272.87 & 3 & 0.00 & 0.24 & 17482 & 639 & 273.00 & 303.80 \\
\hline & & & & & & 4 & 0.00 & 0.27 & 45864 & 784 & 272.74 & 305.05 \\
\hline
\end{tabular}

D2 7

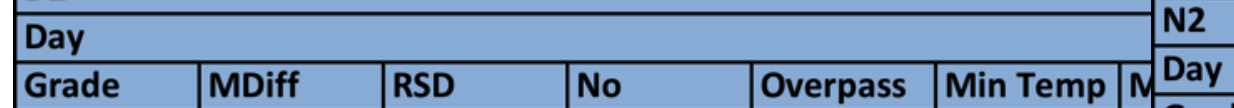

\begin{tabular}{|c|c|c|c|c|c|c|c|c|c|c|c|c|}
\hline irade & |MDiff & RSD & No & Overpass & Min Temp & & & & & & & \\
\hline 1 & 0.04 & 0.22 & 475 & 139 & \begin{tabular}{|l|}
281.21 \\
\end{tabular} & Grade & MDiff & RSD & No & Overpass & Min Temp & Max Temp \\
\hline $2 \mathrm{a}$ & 0.09 & 0.32 & 1550 & 248 & 273.54 & 1 & 0.07 & 0.27 & 817 & 139 & 273.27 & 304.51 \\
\hline $2 \mathrm{~b}$ & 0.09 & 0.28 & 1921 & 233 & 273.62 & $2 \mathrm{a}$ & 0.12 & 0.38 & 2342 & 248 & 273.16 & 304.51 \\
\hline 3 & 0.10 & 0.35 & 5832 & 332 & 273.13 & $2 \mathrm{~b}$ & 0.11 & 0.32 & 3085 & 233 & 273.19 & 304.51 \\
\hline 4 & 0.09 & 0.41 & 17445 & 505 & 272.89 & 3 & 0.14 & 0.40 & 8731 & 332 & 273.14 & 304.51 \\
\hline & & & & & & 4 & 0.11 & 0.44 & 25984 & 505 & 273.02 & 304.77 \\
\hline
\end{tabular}

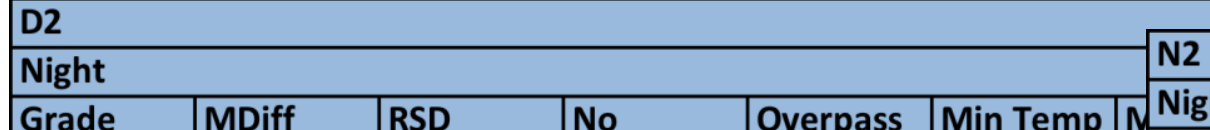

\begin{tabular}{|l|l|l|l|l|l|l|}
\hline Grade & MDiff & RSD & No & Overpass & Min Temp & Night \\
\hline
\end{tabular}

\begin{tabular}{|c|c|c|c|c|c|c|c|c|c|c|c|c|}
\hline Grade & MDiff & RSD & No & Overpass & Min Temp & Night & & & & & & \\
\hline 1 & 0.04 & 0.23 & 1339 & 317 & 273.16 & Grade & MDiff & RSD & No & Overpass & Min Temp & Max Temp \\
\hline $2 a$ & 0.05 & 0.32 & 3051 & 535 & 272.93 & 1 & 0.07 & 0.29 & 1772 & 317 & 273.33 & 304.46 \\
\hline $2 b$ & 0.03 & 0.24 & 4775 & 457 & 273.01 & $2 a$ & 0.04 & 0.39 & 4535 & 535 & 272.99 & 304.46 \\
\hline 3 & 0.05 & 0.33 & 11846 & 639 & 272.93 & $2 \mathrm{~b}$ & 0.05 & 0.30 & 6739 & 457 & 273.32 & 304.53 \\
\hline 4 & 0.06 & 0.36 & 31362 & 784 & 272.71 & 3 & 0.04 & 0.39 & 17482 & 639 & 272.99 & 304.53 \\
\hline & & & & & & 4 & 0.04 & 0.42 & 45864 & 784 & 272.72 & 305.73 \\
\hline
\end{tabular}




\section{Validation results - D3,D2}

- Grade 2b, CV5, same pixel, night, day

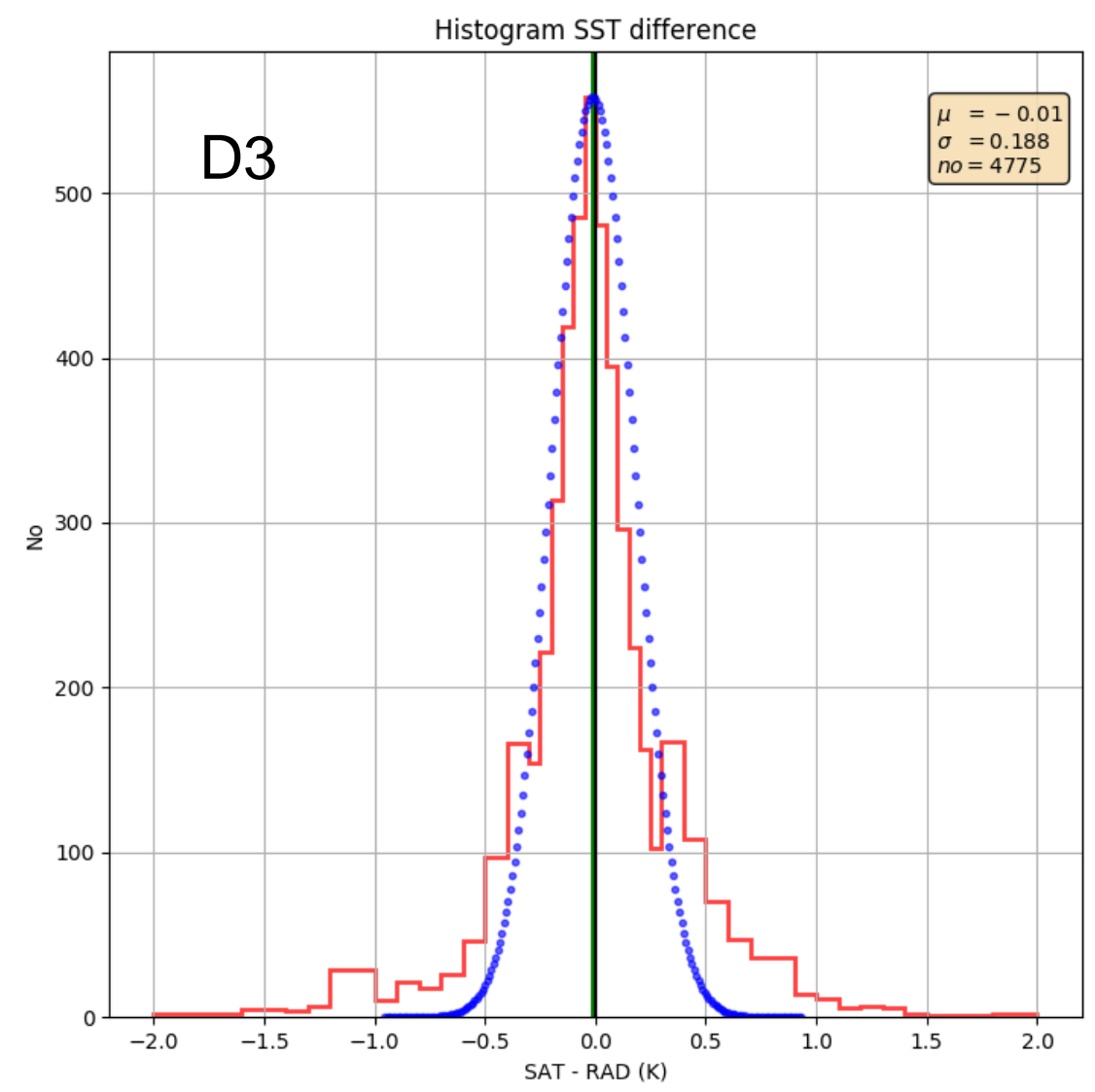

s4sstrli1, all, sstdiff_D3, grade 2b, night, ghrsst-5, qi-all - HuberT

processed 20200509 (c) 2020 ISAR team - v5.2 ships4sst: FRM4SST

GHRSST XXI

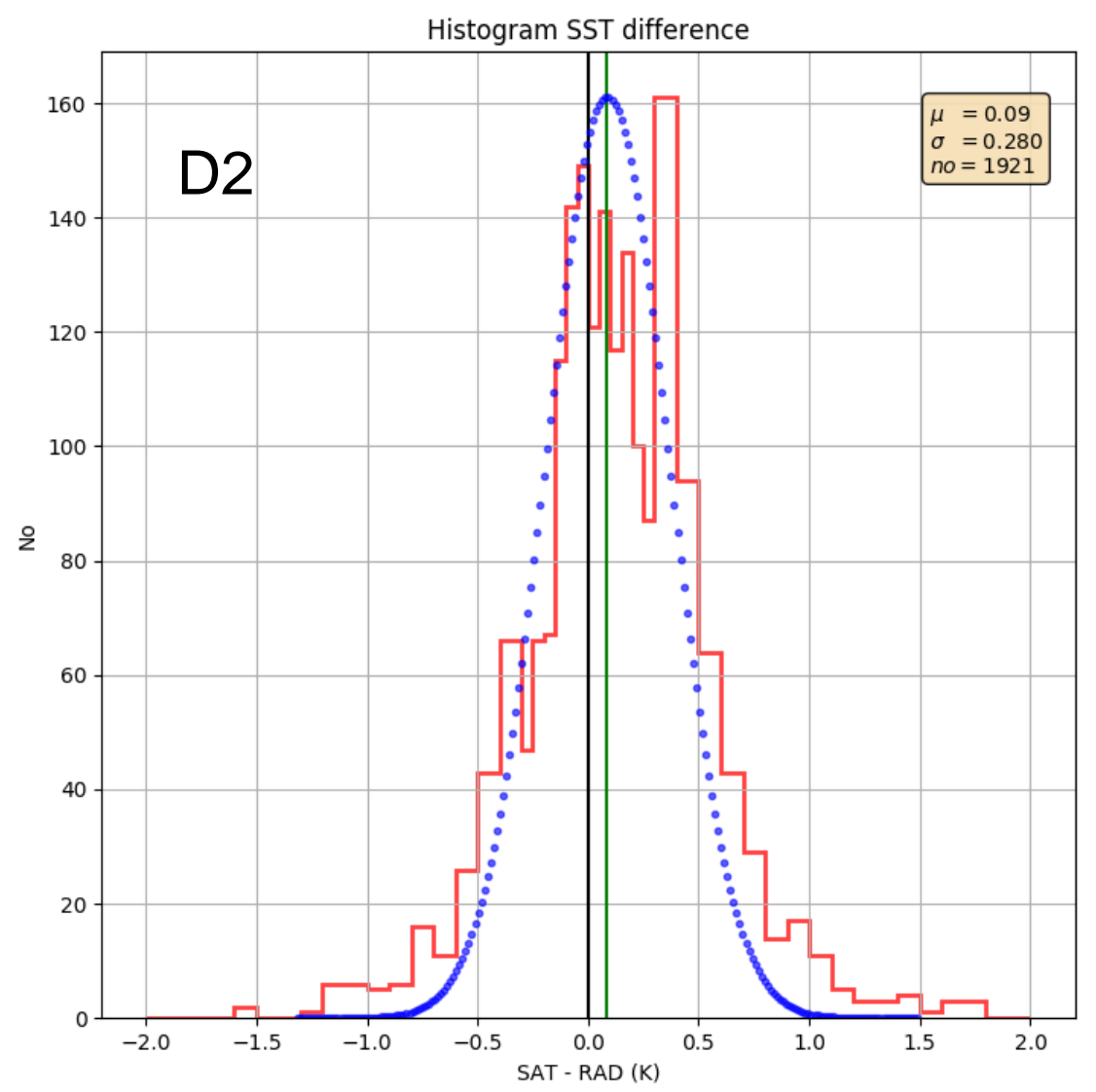

54sstr1i1, all, sstdiff_D2, grade 2b, day, ghrsst-5, qi-all - Huber

processed 20200509 (c) 2020 ISAR team - v5.2

\section{Cesa} 02 June 2020

Page 20

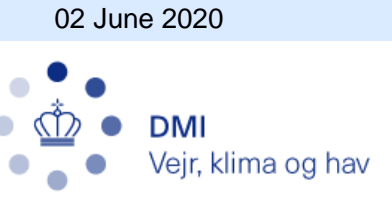

Space 


\section{Validation results - D3,D2}

- Grade 2b, CV5, same pixel, both night

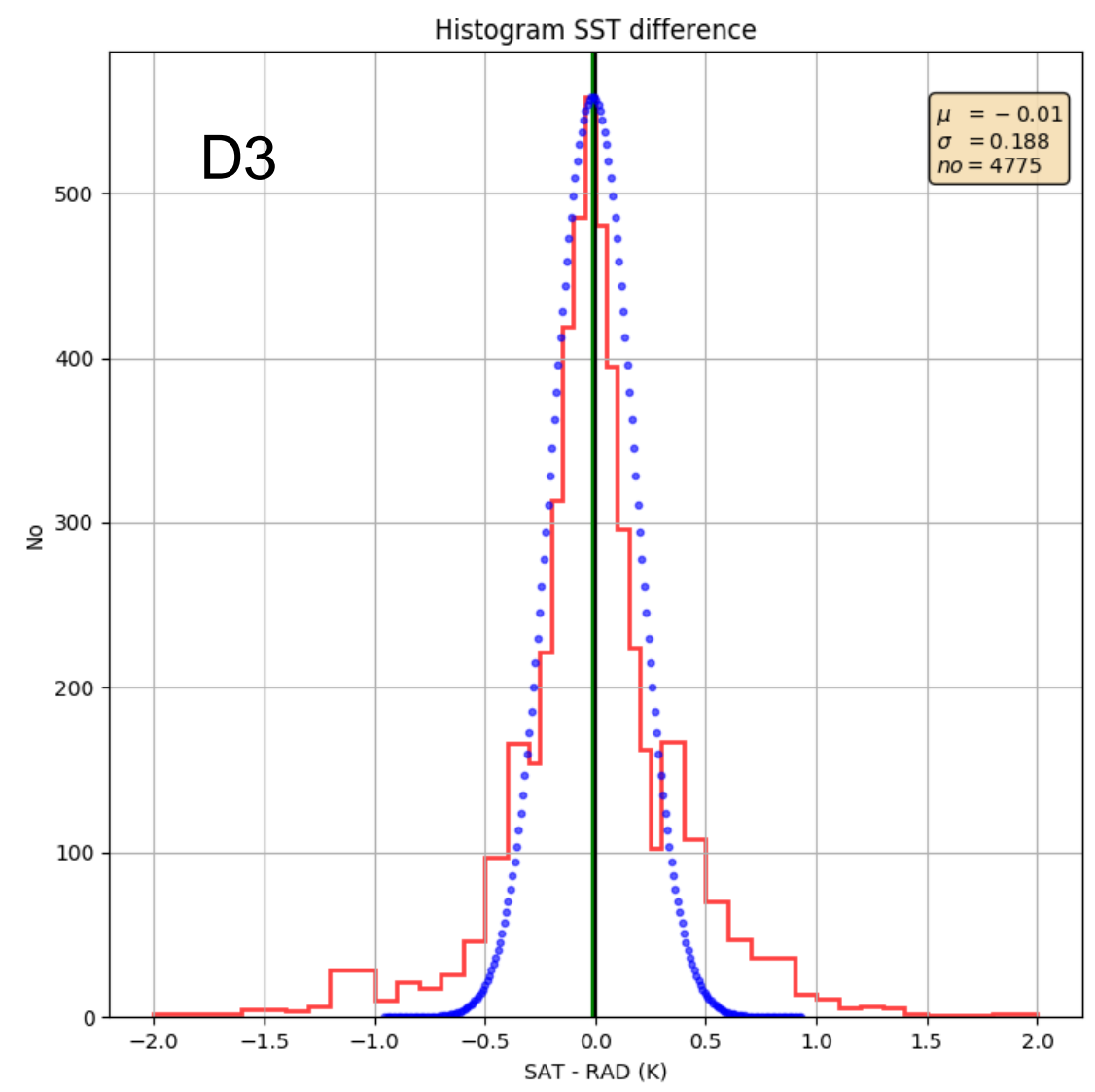

54sstr1i1, all, sstdiff_D3, grade 2b, night, ghrsst-5, qi-all - HuberT

processed 20200509 (c) 2020 ISAR team - v5.2

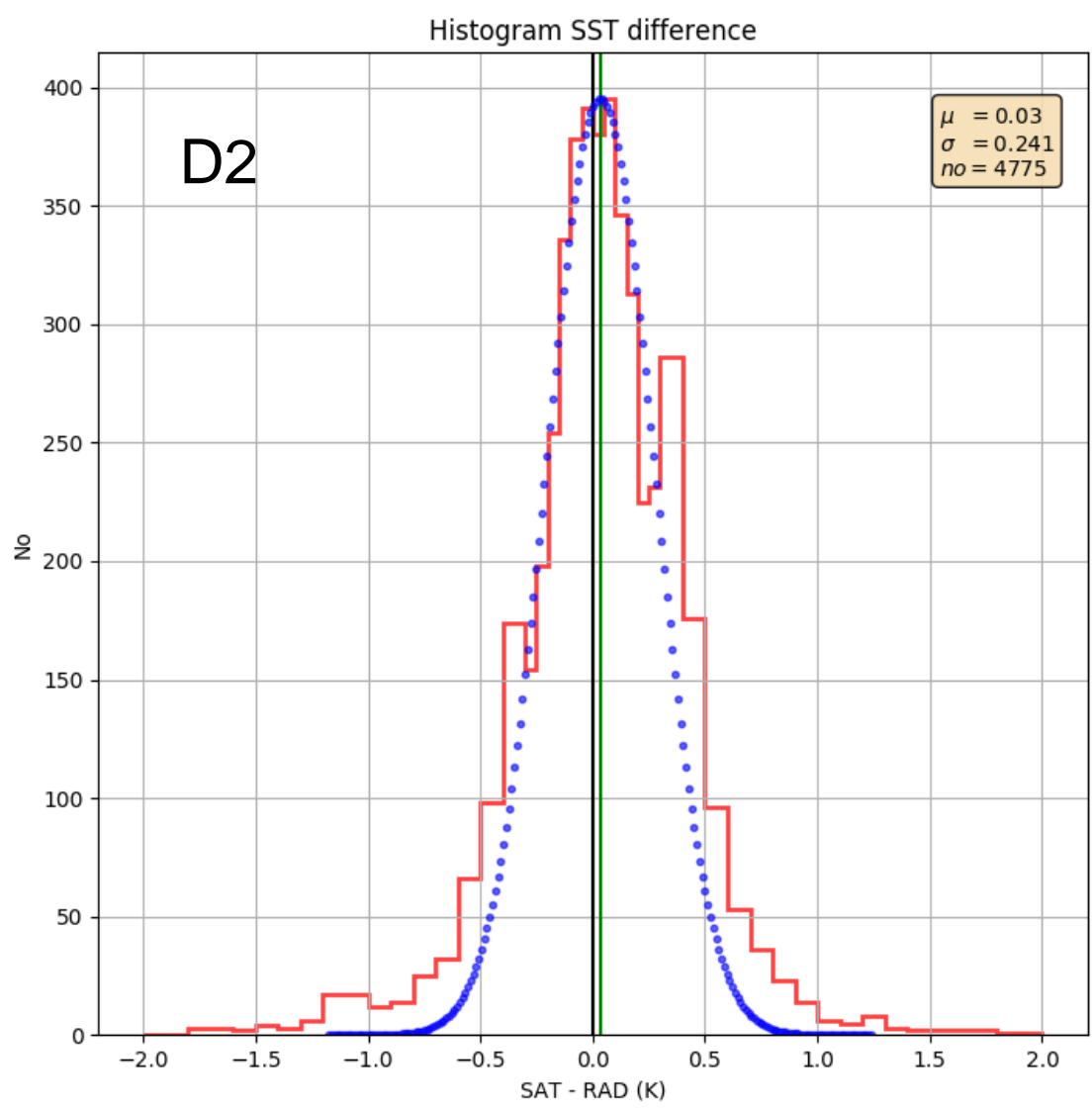

s4sstr1i1, all, sstdiff_D2, grade 2b, night, ghrsst-5, qi-all - HuberT

processed 20200509 (c) 2020 ISAR team - v5.2 02 June 2020

Page 21 


\section{Validation results $-\mathrm{N} 3, \mathrm{~N} 2$}

- Grade 2b, CV5, same pixel, night, day

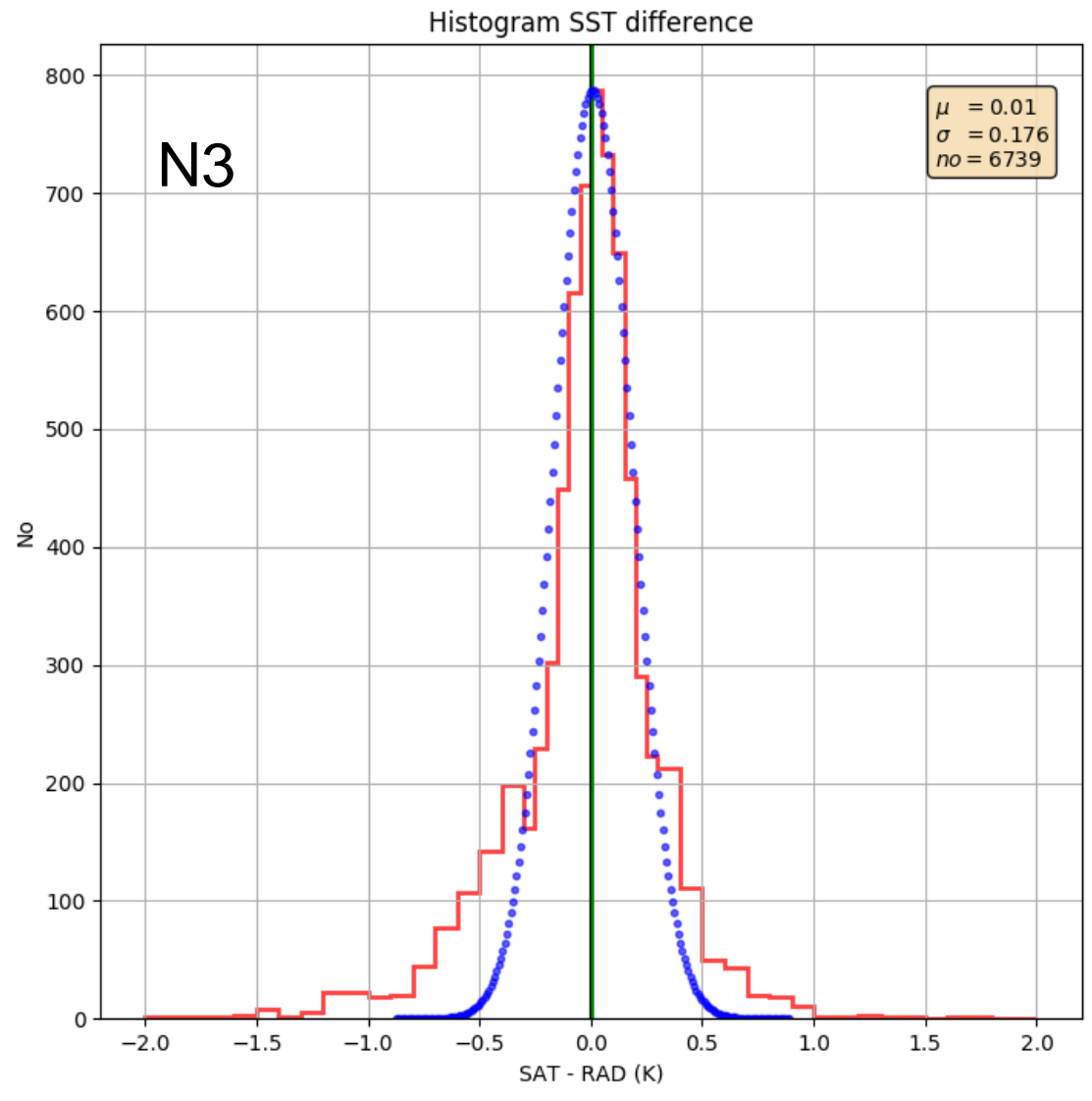

54sstr1i1, all, sstdiff_N3, grade 2b, night, ghrsst-5, qi-all - HuberT

processed 20200509 (c) 2020 ISAR team - v5.2

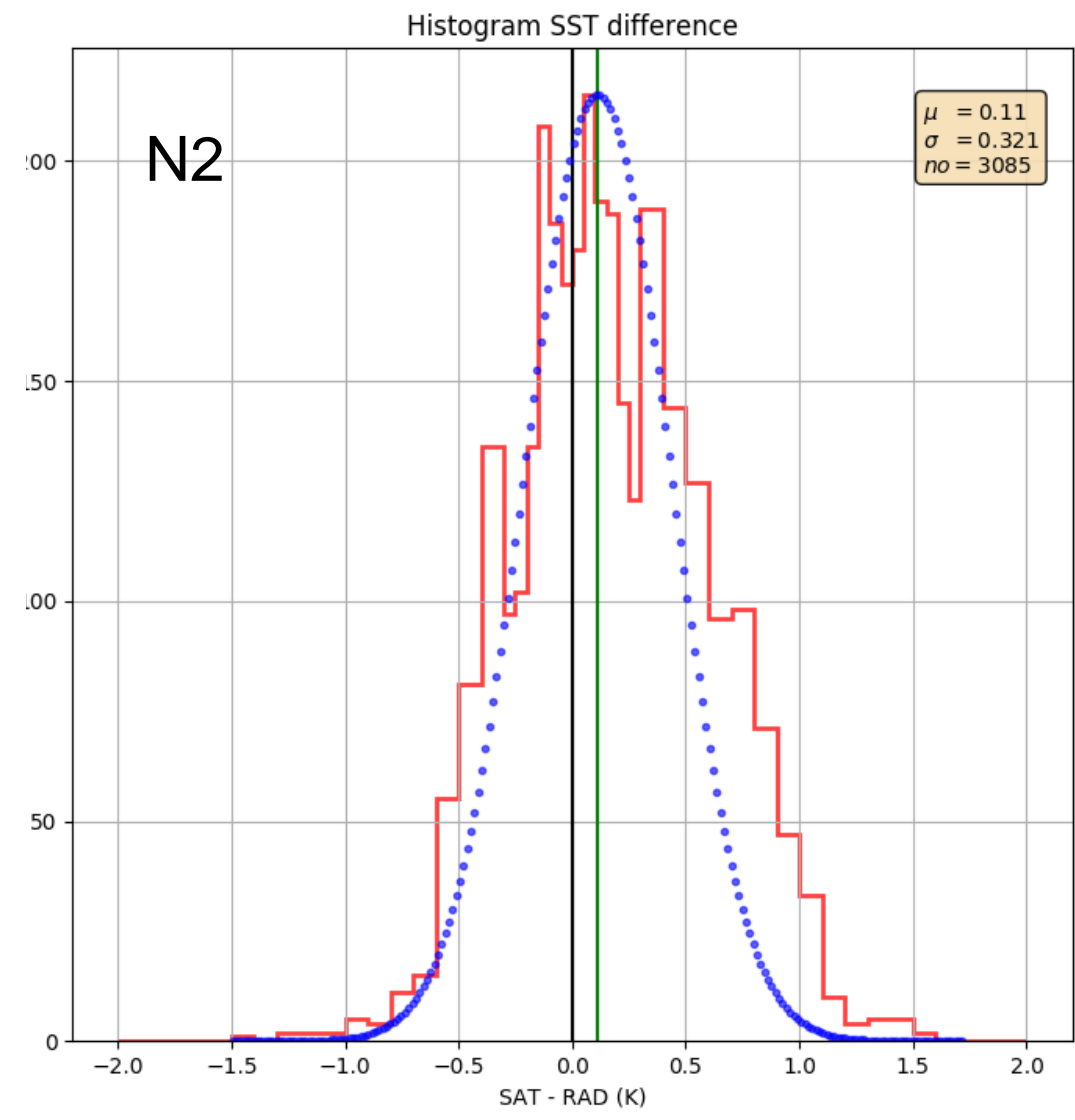

54sstr1i1, all, sstdiff_N2, grade 2b, day, ghrsst-5, qi-all - HuberT processed 20200509 (c) 2020 ISAR team - v5.2 


\section{Validation results $-\mathrm{N} 3, \mathrm{~N} 2$}

- Grade 2b, CV5, same pixel, both night

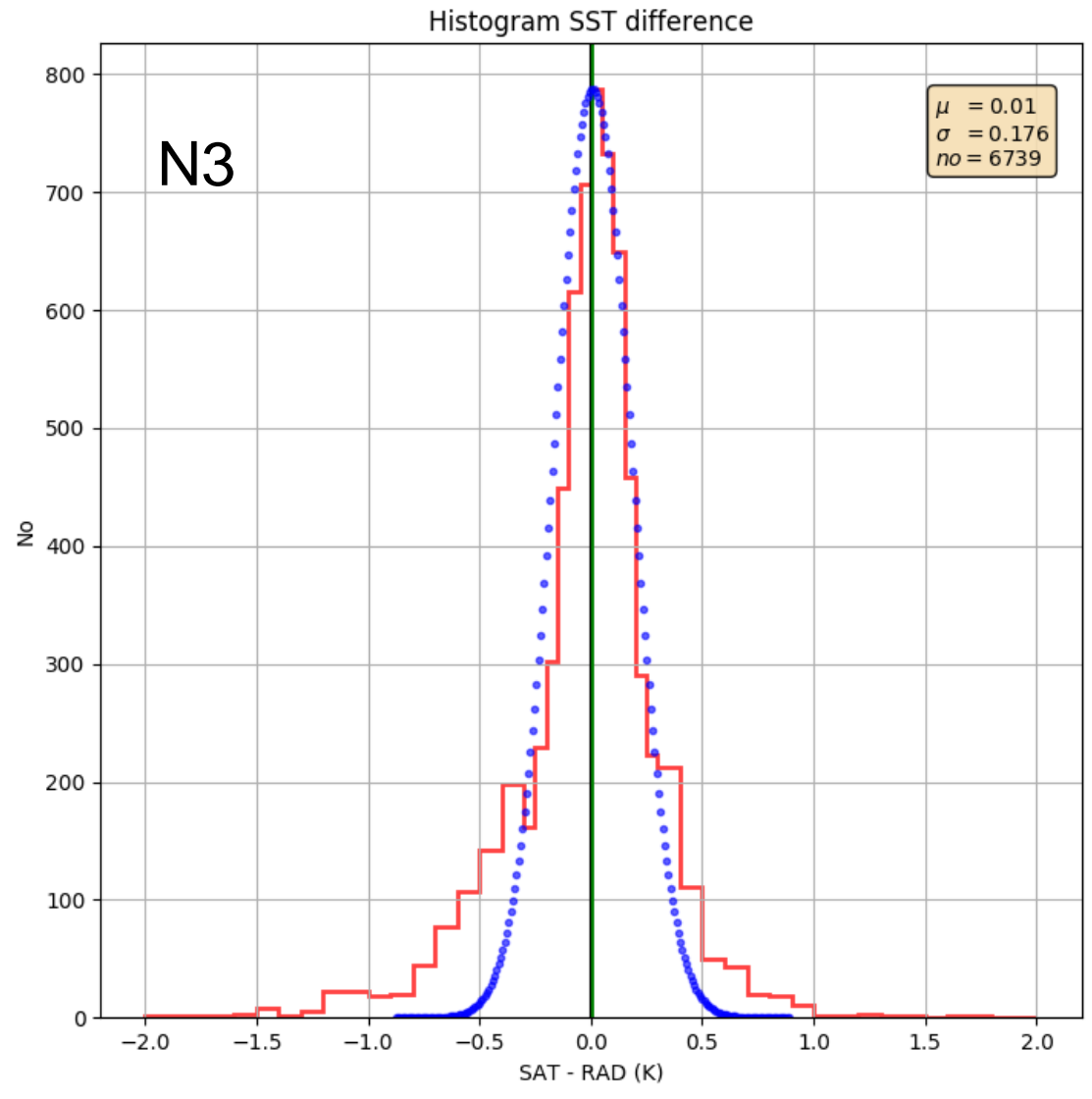

s4sstr1i1, all, sstdiff_N3, grade 2b, night, ghrsst-5, qi-all - HuberT

processed 20200509 (c) 2020 ISAR team - v5.2

ships4sst: FRM4SST

GHRSST XXI

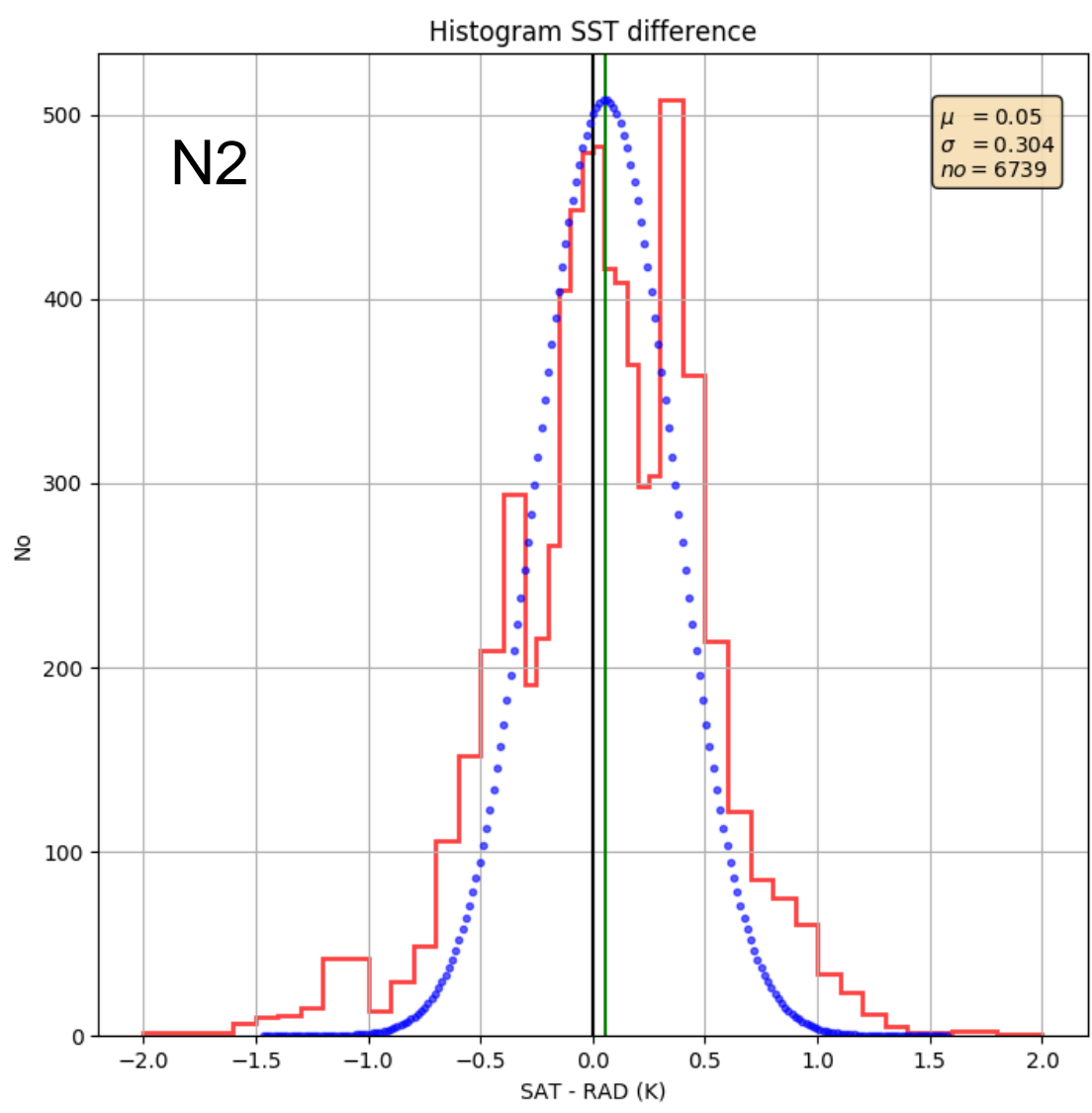

s4sstr1i1, all, sstdiff_N2, grade 2b, night, ghrsst-5, qi-all - HuberT

processed 20200509 (c) 2020 ISAR team - v5.2

Page 23 02 June 2020

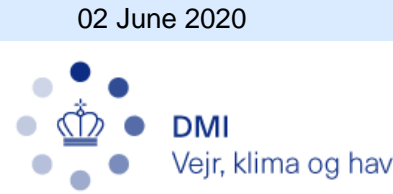




\section{Conclusions}

- Validation of SLSTR with FRM data:

- Shows very good results for SLSTR

- Virtually no mean difference at night and only a small difference at day time

- RSD is lower at night than day time and comparable to AATSR .

- QI results

- Need checking why so few QI 5

- Need some work to investigate the differences between AATSR and SLSTR results:

- Thresholds too stringent/wrong for SLSTR?

- Developed for one region (English Channel and Bay of Biscay, maybe not applicable globally?

- Processing differences? 


\section{What else is available}

- NRT results for 2018, in appendix

- Results for GHRSST CV 3 and 4

- Dependence plots for WST, D3, D2, N3 and N2:

- Latitude, Wind, uncertainty

- Temporal match-up window

- Spatial match-up window

- QI method on D3, D2, N3 and N2

- Regional results for each route, split by ships name. The have the same set of statistics and plots as the global results 


\section{Validation results - global}

\section{- NRT- April 2018 - Dec 2018}

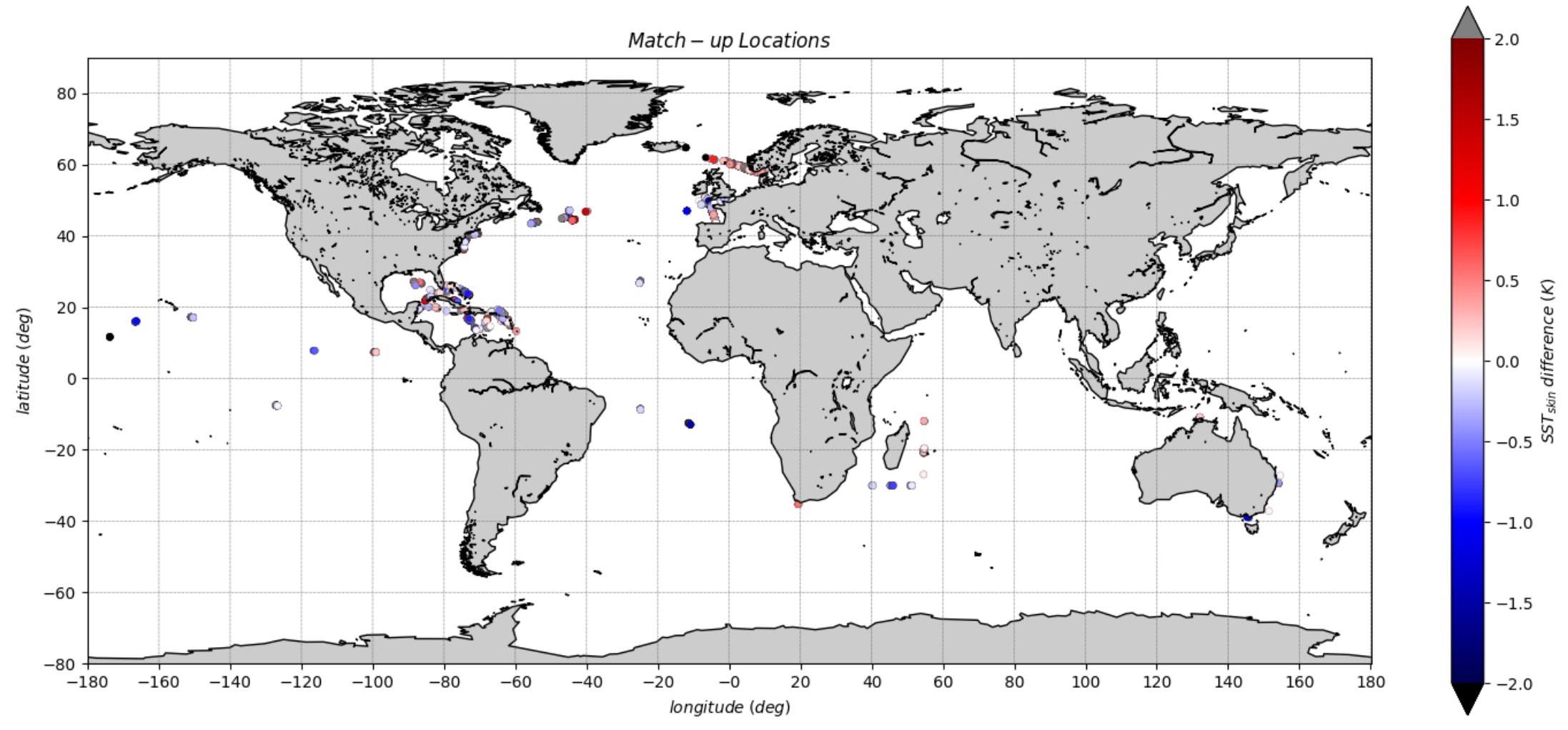

\section{- esa}


Validation results global histograms NRT: 2018

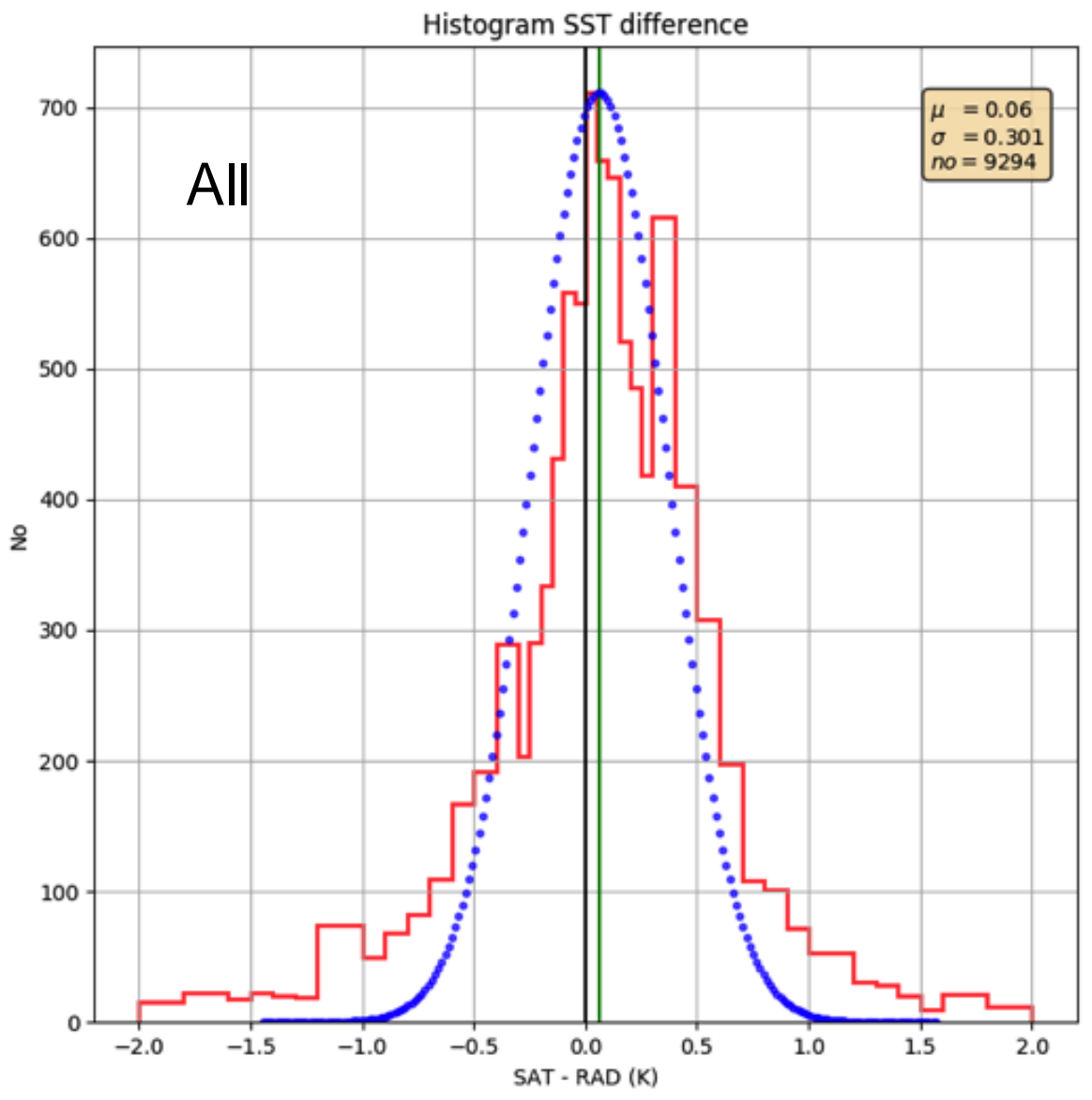

s4sstr1i1, all, sstdiff _sst_wst, grade 2b, all, ghrsst-all, qi-all - HuberT processed 20200508 (c) 2020 ISAR team - v5.2

\section{- cesa}
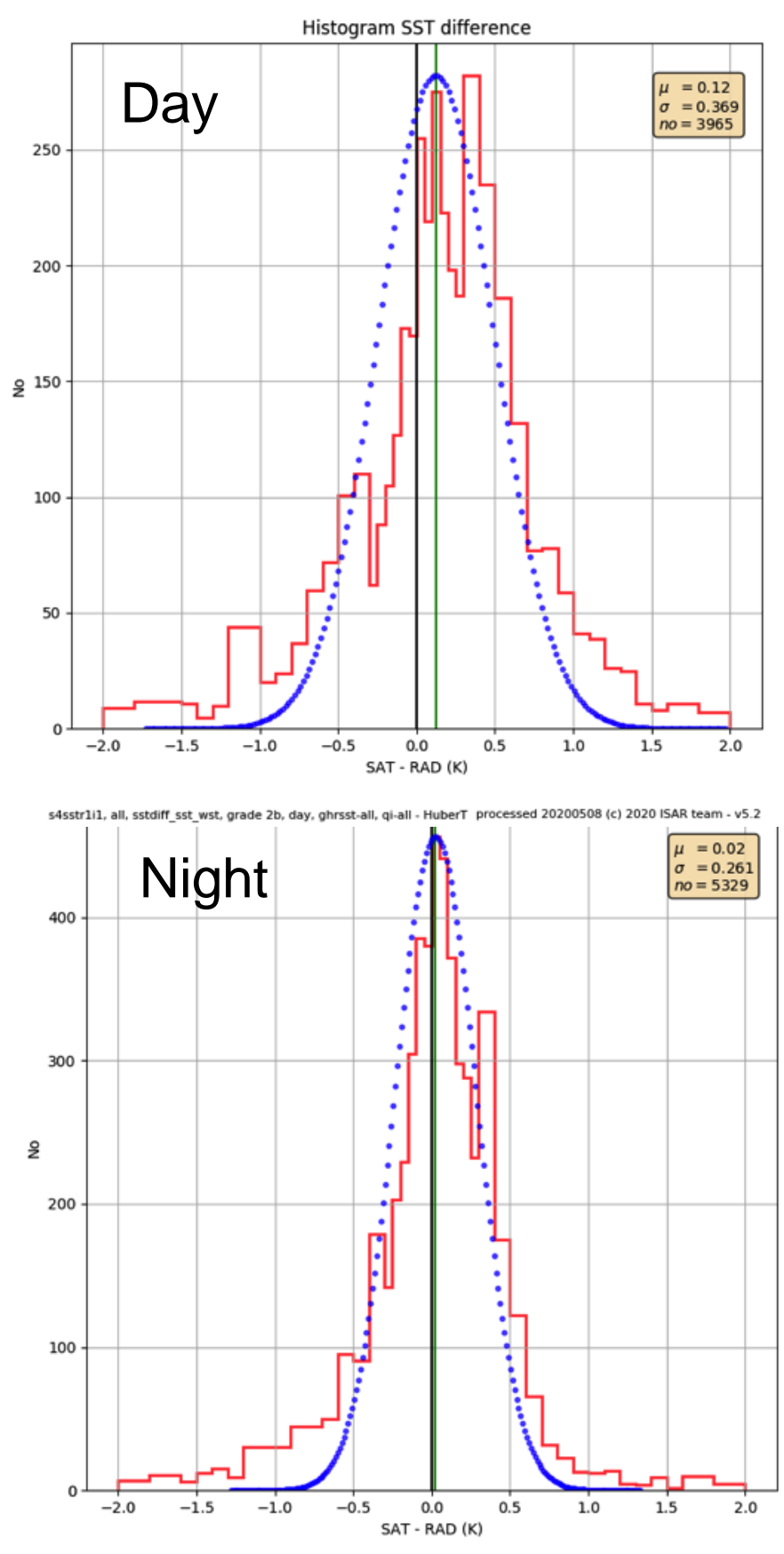


\section{Histograms - CV5, All}

NRT: 2018

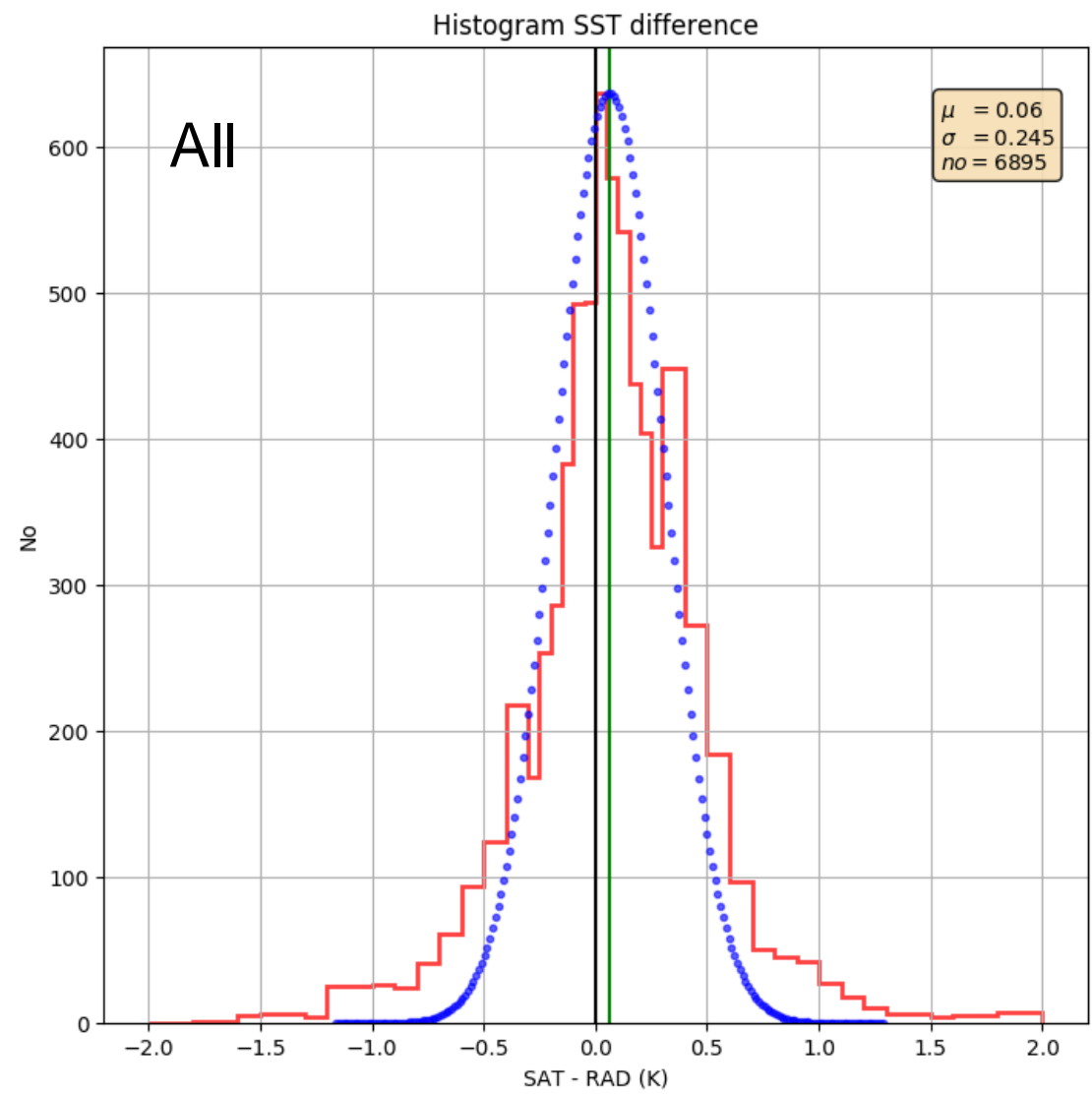

s4sstr1i1, all, sstdiff_sst_wst, grade 2b, all, ghrsst-5, qi-all - HuberT

processed 20200511 (c) 2020 ISAR team - v5.2
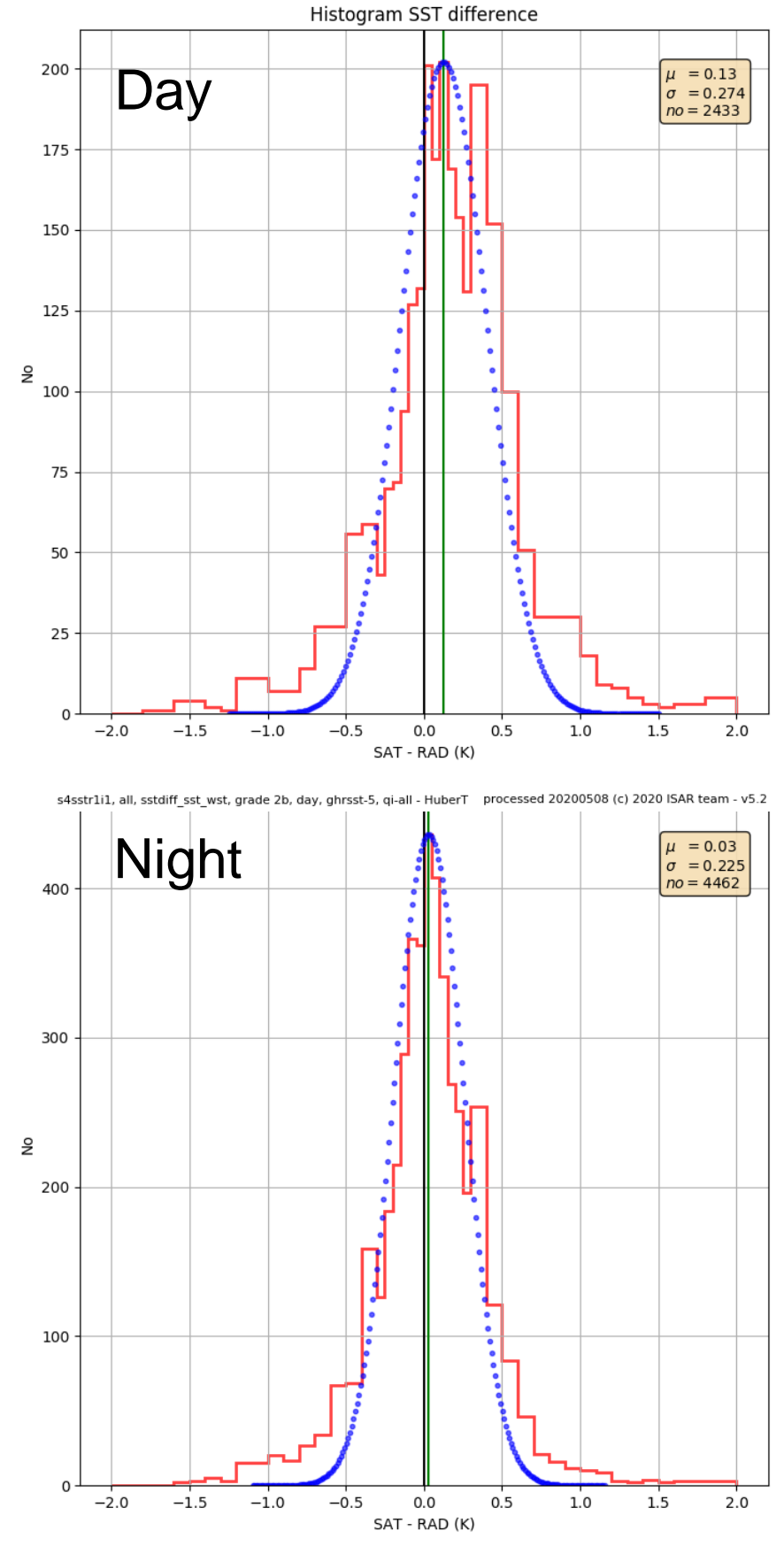


\section{Validation scatter - all}

NRT: 2018

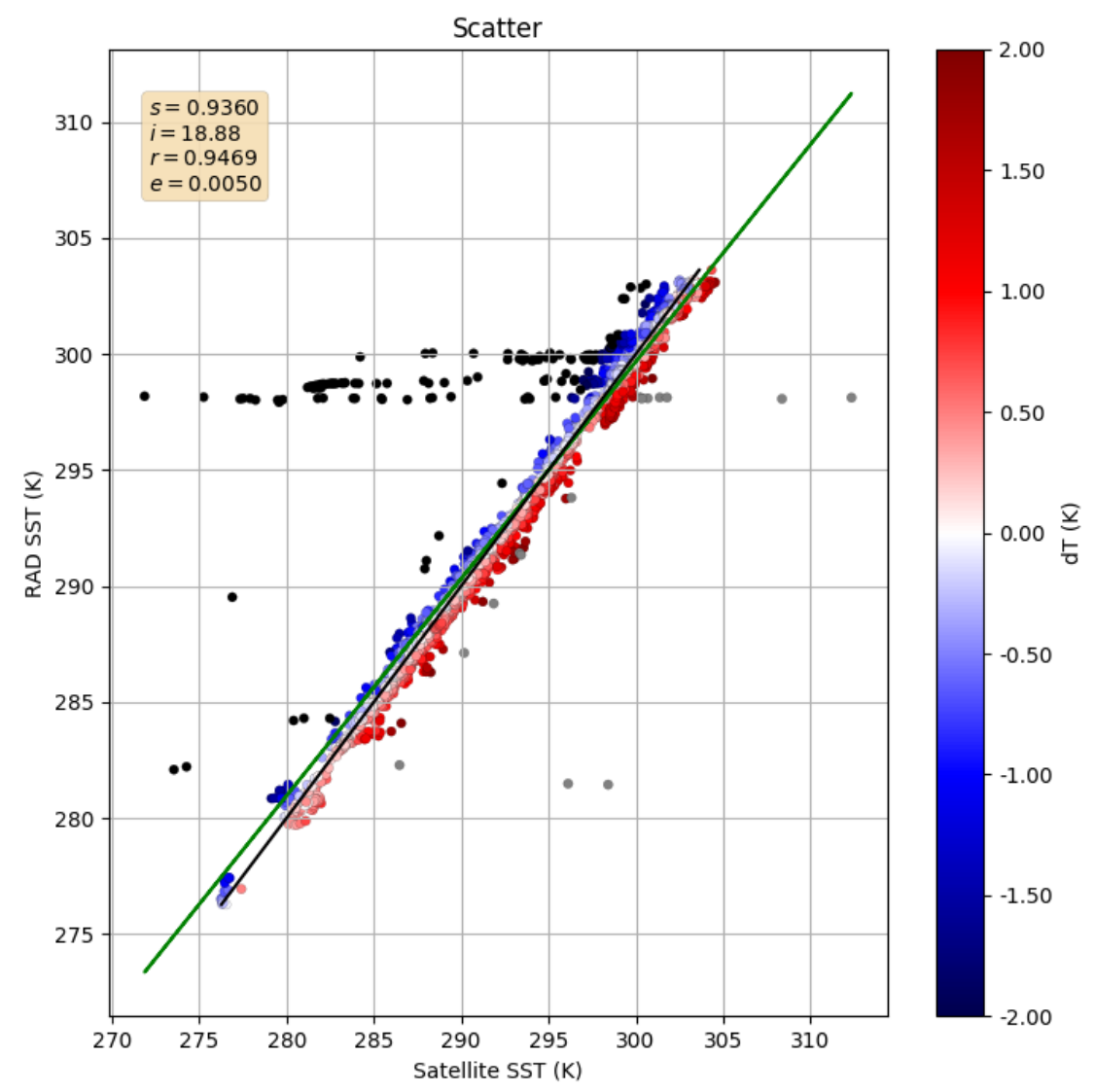

s4sstr1i1, all, sstdiff_sst_wst, grade 2b, day, ghrsst-all, qi-all - HuberT processed 20200508 (c) 2020 ISAR team - v5.2

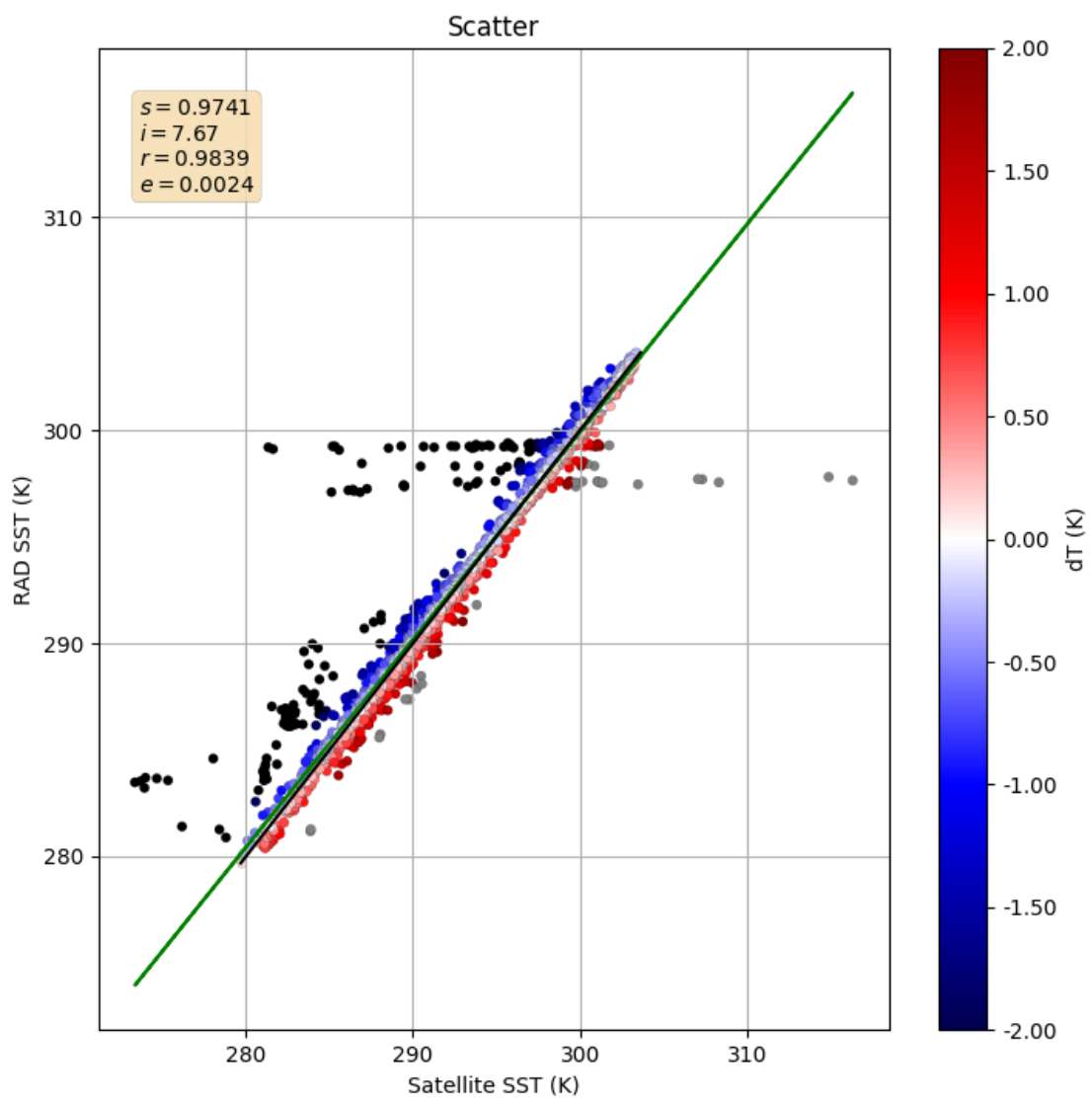

s4sstr1i1, all, sstdiff_sst_wst, grade 2b, night, ghrsst-all, qi-all - HuberTprocessed 20200508 (c) 2020 ISAR team - v5.2 


\section{Validation - scatter, CV 5}

NRT: 2018

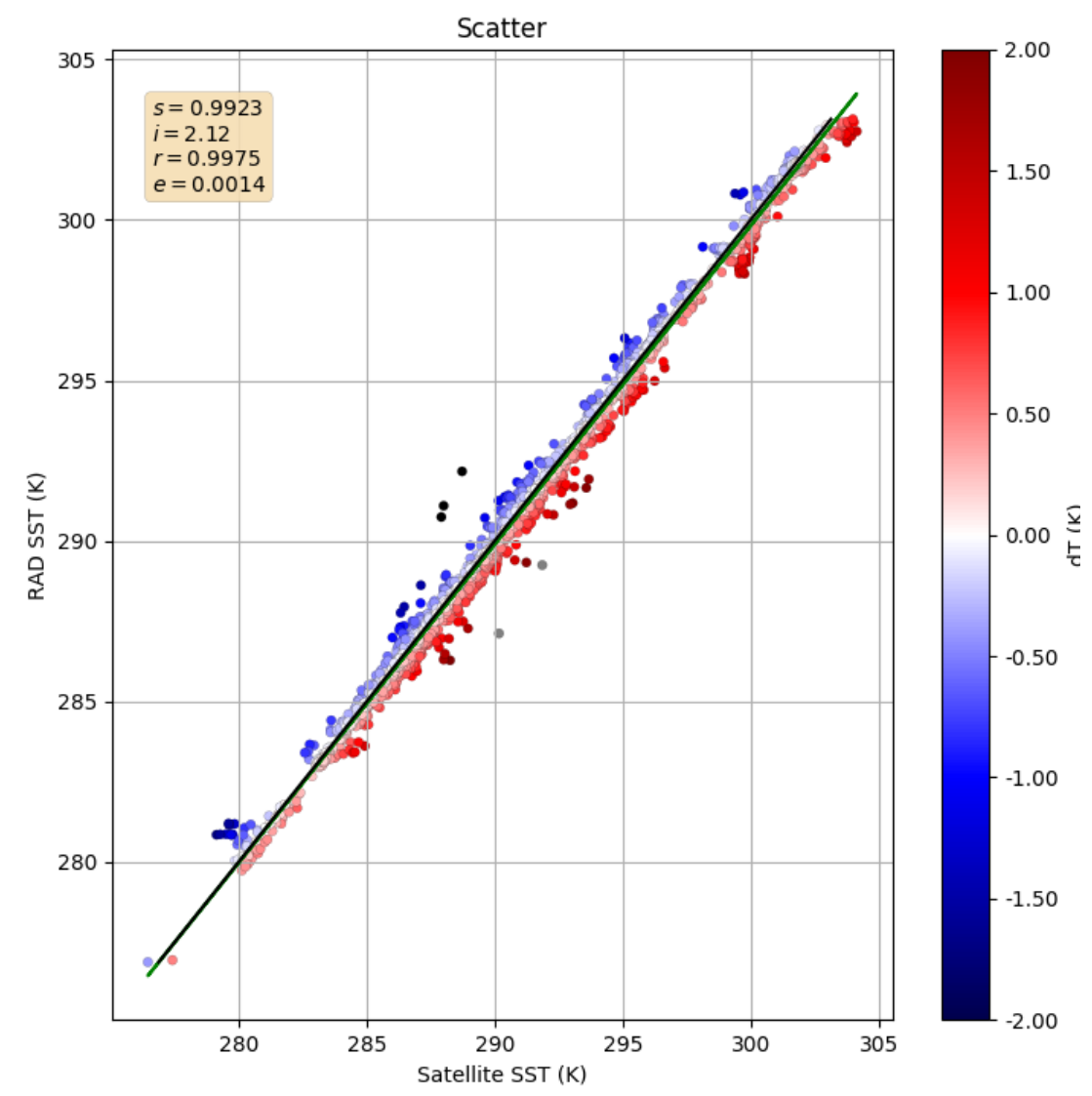

54sstrii1, all, sstdiff_sst_wst, grade 2b, day, ghrsst-5, qi-all - HuberT processed 20200508 (c) 2020 ISAR team - v5.:

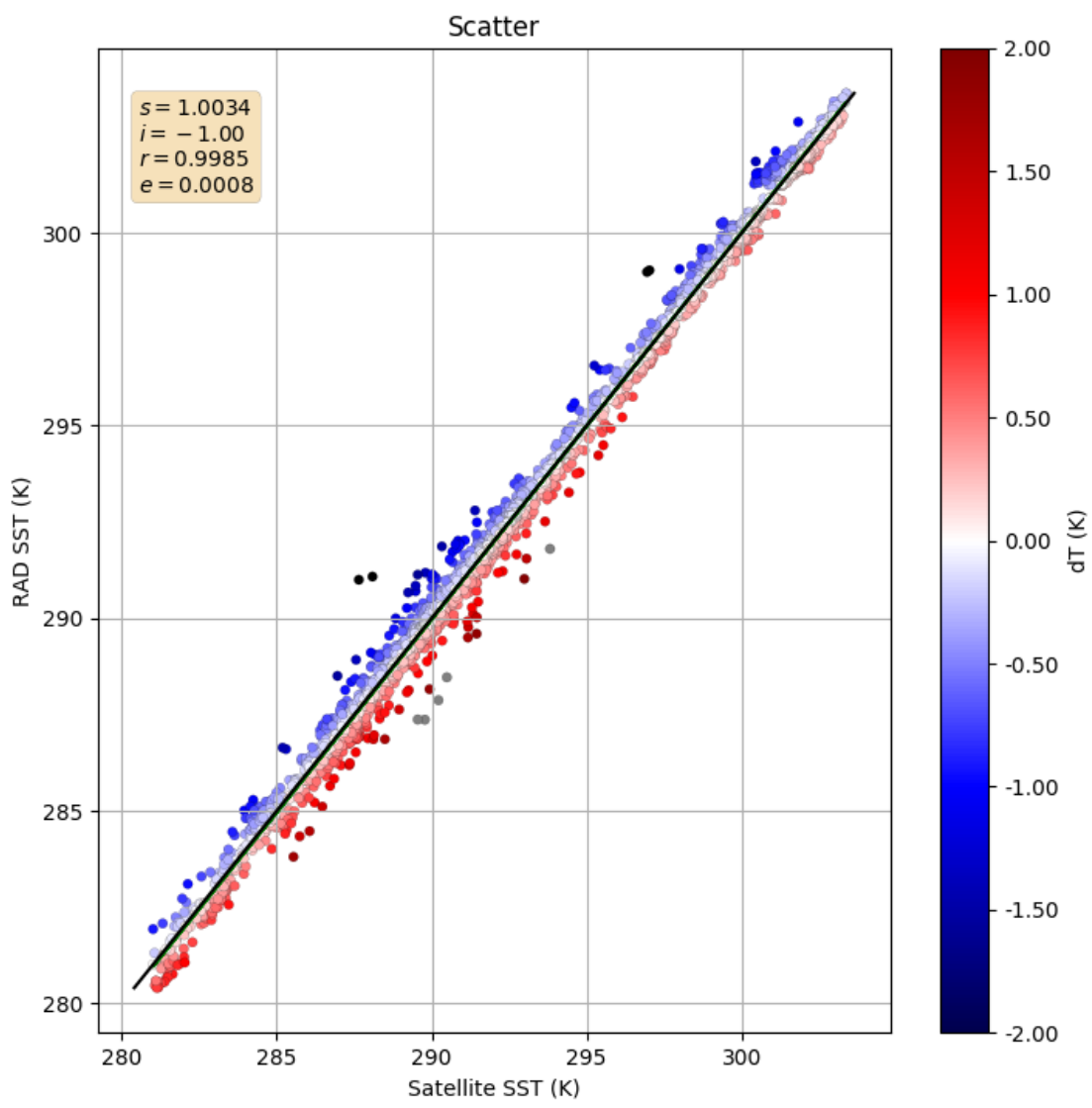

54sstr1i1, all, sstdiff_sst_wst, grade 2b, night, ghrsst-5, qi-all - HuberT processed 20200508 (c) 2020 ISAR team - v5.2 
Validation results - statistics

- NRT: 2018

\begin{tabular}{|r|r|r|r|r|r|r|}
\hline \multicolumn{1}{|l|}{ lay } \\
\hline \multicolumn{1}{|l|}{ Mrade } & MDiff & RSD & \multicolumn{1}{l|}{ No } & Overpass & Min Temp & Max Temp \\
\hline 1 & 0.09 & 0.29 & 1040 & 117 & 279.51 & 304.03 \\
\hline $2 \mathrm{a}$ & 0.04 & 0.43 & 3202 & 224 & 270.45 & 305.87 \\
\hline $\mathbf{2 b}$ & $\mathbf{0 . 1 2}$ & $\mathbf{0 . 3 7}$ & $\mathbf{3 9 6 5}$ & $\mathbf{1 8 7}$ & $\mathbf{2 7 1 . 8 8}$ & $\mathbf{3 1 2 . 3 4}$ \\
\hline 3 & 0.05 & 0.49 & 12219 & 295 & 270.18 & 313.67 \\
\hline 4 & 0.02 & 0.60 & 33461 & 384 & 270.18 & 317.94 \\
\hline
\end{tabular}

\begin{tabular}{|r|r|r|r|r|r|r|}
\hline \multicolumn{9}{|l|}{ WST } \\
\hline \multicolumn{10}{|l|}{ ALL } \\
\hline Grade & \multicolumn{1}{|l|}{ MDiff } & \multicolumn{1}{|l|}{ RSD } & No & Overpass & Min Temp & Max Temp \\
\hline 1 & 0.08 & 0.26 & 2457 & 269 & 279.51 & 304.03 \\
\hline $2 \mathrm{a}$ & 0.03 & 0.37 & 6975 & 490 & 270.45 & 305.87 \\
\hline $\mathbf{2 b}$ & $\mathbf{0 . 0 6}$ & $\mathbf{0 . 3 0}$ & $\mathbf{9 2 9 4}$ & $\mathbf{4 1 6}$ & $\mathbf{2 7 1 . 8 8}$ & $\mathbf{3 1 6 . 3 4}$ \\
\hline 3 & 0.02 & 0.40 & 26753 & 609 & 270.18 & 316.34 \\
\hline 4 & -0.01 & 0.47 & 71568 & 754 & 270.18 & 317.94 \\
\hline
\end{tabular}

\begin{tabular}{|r|r|r|r|r|r|r|}
\hline \multicolumn{1}{|l|}{ Night } \\
\hline Grade & \multicolumn{1}{|l|}{ MDiff } & \multicolumn{1}{l|}{ RSD } & No & Overpass & Min Temp & Max Temp \\
\hline 1 & 0.06 & 0.24 & 1417 & 152 & 279.77 & 303.23 \\
\hline $2 \mathrm{a}$ & 0.03 & 0.33 & 3773 & 266 & 270.82 & 303.47 \\
\hline $\mathbf{2 b}$ & $\mathbf{0 . 0 2}$ & $\mathbf{0 . 2 6}$ & $\mathbf{5 3 2 9}$ & $\mathbf{2 2 9}$ & $\mathbf{2 7 3 . 3 5}$ & $\mathbf{3 1 6 . 3 4}$ \\
\hline 3 & -0.01 & 0.34 & 14534 & 315 & 270.60 & 316.34 \\
\hline 4 & -0.03 & 0.38 & 38107 & 376 & 270.54 & 317.94 \\
\hline
\end{tabular}




\section{Validation results - statistics}

- NRT: 2018 - CV 5

\begin{tabular}{|r|r|r|r|r|r|r|}
\hline \multicolumn{1}{|l|}{ Day } \\
\hline \multicolumn{1}{|l|}{ Grade } & \multicolumn{1}{l|}{ MDiff } & \multicolumn{1}{l|}{ RSD } & No & Overpass & Min Temp & Max Temp \\
\hline 1 & 0.09 & 0.21 & 649 & 74 & 279.51 & 304.03 \\
\hline $2 \mathrm{a}$ & 0.11 & 0.30 & 1844 & 144 & 274.94 & 305.13 \\
\hline $\mathbf{2 b}$ & $\mathbf{0 . 1 3}$ & $\mathbf{0 . 2 7}$ & $\mathbf{2 4 3 3}$ & $\mathbf{1 3 8}$ & $\mathbf{2 7 6 . 4 6}$ & $\mathbf{3 0 4 . 1 4}$ \\
\hline 3 & 0.11 & 0.36 & 7018 & 205 & 274.94 & 305.13 \\
\hline 4 & 0.09 & 0.46 & 18957 & 287 & 274.70 & 305.37 \\
\hline
\end{tabular}

\begin{tabular}{|r|r|r|r|r|r|r|}
\hline \multicolumn{10}{|l|}{ WST } \\
\hline \multicolumn{10}{|l|}{ ALL } \\
\hline Grade & \multicolumn{1}{|l|}{ MDiff } & RSD & No & Overpass & Min Temp & Max Temp \\
\hline 1 & 0.07 & 0.21 & 1827 & 205 & 279.51 & 304.03 \\
\hline $2 \mathrm{a}$ & 0.06 & 0.29 & 4984 & 374 & 274.94 & 305.13 \\
\hline $\mathbf{2 b}$ & $\mathbf{0 . 0 6}$ & $\mathbf{0 . 2 4}$ & $\mathbf{6 8 9 5}$ & $\mathbf{3 3 9}$ & $\mathbf{2 7 6 . 4 6}$ & $\mathbf{3 0 4 . 1 4}$ \\
\hline 3 & 0.04 & 0.32 & 19049 & 481 & 273.56 & 305.13 \\
\hline 4 & 0.02 & 0.38 & 50565 & 624 & 273.56 & 305.57 \\
\hline
\end{tabular}

\begin{tabular}{|c|c|c|c|c|c|c|}
\hline \\
\hline \multicolumn{7}{|c|}{\begin{tabular}{|l|l} 
Min Temp & Max Temp
\end{tabular}} \\
\hline 1 & 0.06 & 0.21 & 1178 & 131 & 281.07 & 303.23 \\
\hline $2 a$ & 0.03 & 0.29 & 3140 & 230 & 276.67 & 303.47 \\
\hline $2 b$ & 0.03 & 0.23 & 4462 & 201 & 281.01 & 303.39 \\
\hline 3 & 0.00 & 0.30 & 12031 & 276 & 273.56 & 303.50 \\
\hline 4 & -0.02 & 0.33 & 31608 & 337 & 273.56 & 305.57 \\
\hline
\end{tabular}




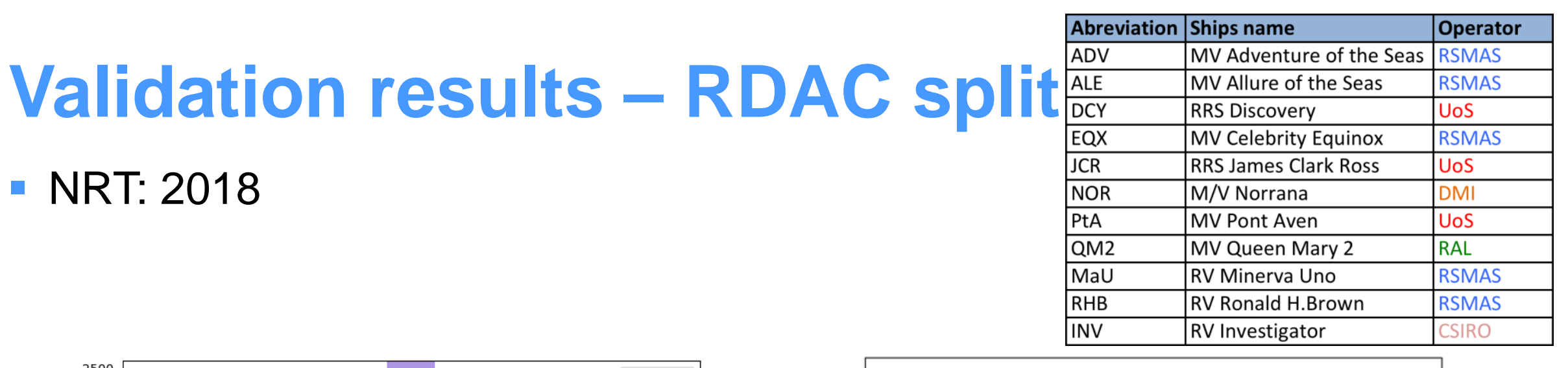

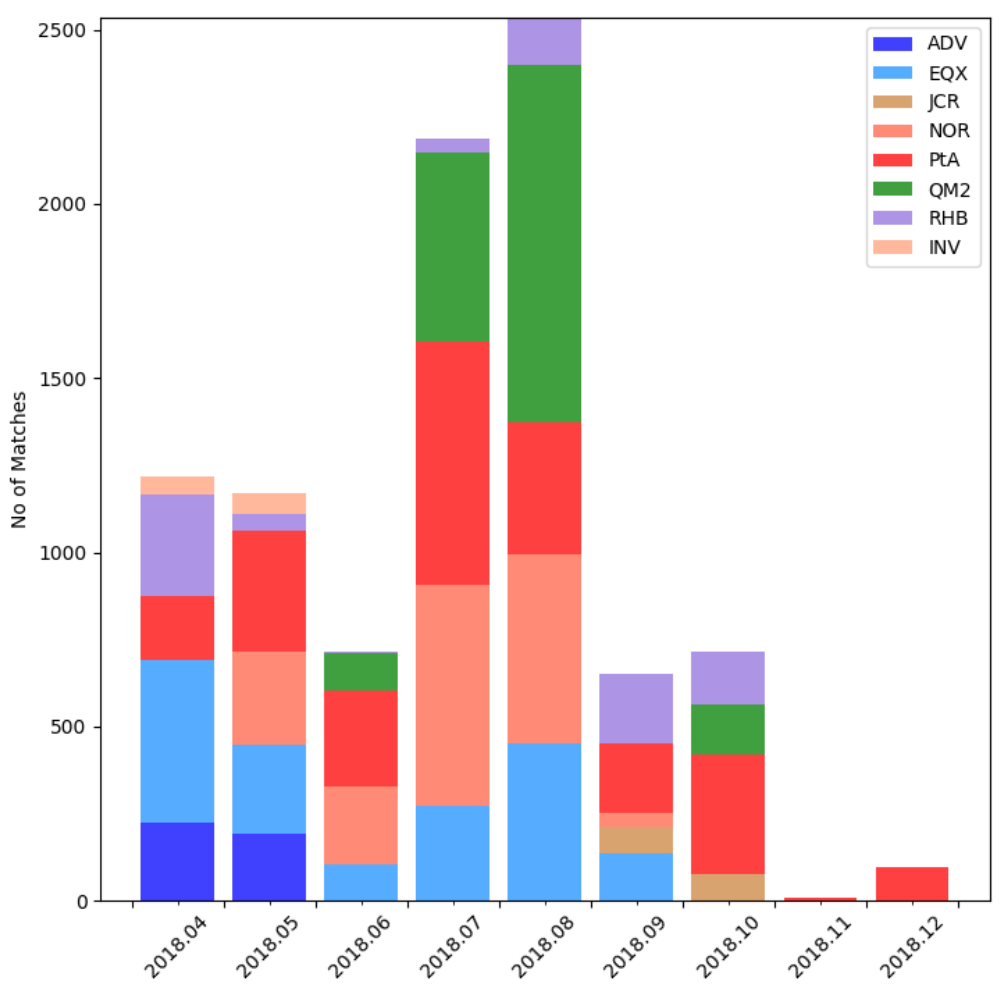

all, sstdiff__sst_wst, grade 2b, all, ghrsst-all - HuberT

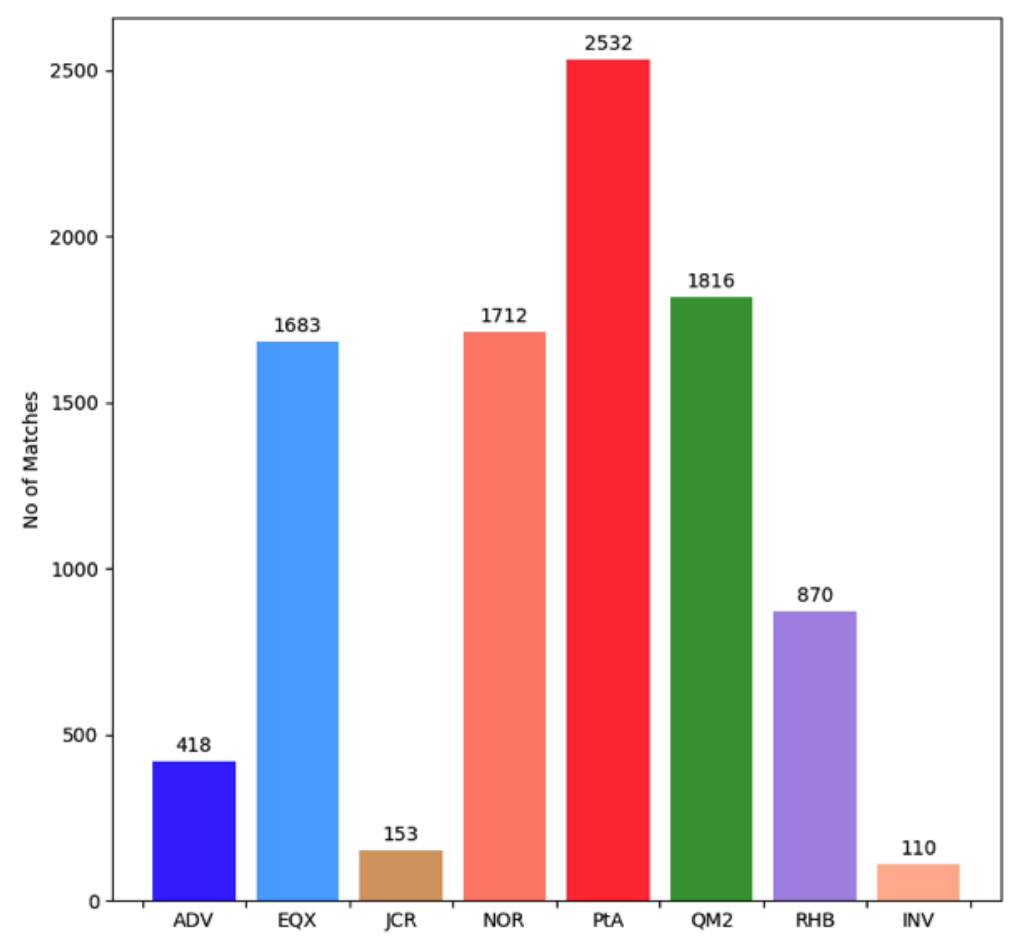

all, sstdiff_sst_wst, grade 2b, all, ghrsst-all - HuberT 